\title{
Extreme Points and Strongly Extreme Points in Orlicz Spaces Equipped with the Orlicz Norm
}

\author{
Y. Cui, H. Hudzik and R. Płuciennik
}

\begin{abstract}
Criteria for extreme points and strongly extreme points of the unit ball in Orlicz spaces with the Orlicz norm are given. These results are applied to characterization of extreme points of $B\left(L^{1}+L^{\infty}\right)$ which corresponds with the result obtained by R. Grza̧ślewicz and H. Schaefer [10] and H. Schaefer [25]. Moreover, we show that every extreme point of $B\left(L^{1}+L^{\infty}\right)$ is strongly extreme. We also get criteria for extreme points of $B\left(L^{p} \cap L^{\infty}\right) \quad(1 \leq p<\infty)$ using Theorem 1 and for strongly extreme points of $B\left(L^{p} \cap L^{\infty}\right) \quad(1 \leq p<\infty)$ applying Theorem 2. Although, criteria for extreme points of $B\left(L^{1} \cap L^{\infty}\right)$ were known (see [13]), we can easily deduce them from our main results and we can extend those results to establish which among extreme points are strongly extreme. The descriptions of the extreme and strongly extreme points of $B\left(L^{p} \cap L^{\infty}\right) \quad(1<p<\infty)$ are original. Moreover, criteria for extreme points and strongly extreme points of the unit ball in the subspace of finite elements of an Orlicz space are deduced on the basis of our main results.
\end{abstract}

Keywords: Orlicz space, Orlicz norm, extreme point, strongly extreme point

AMS subject classification: 46E30, 46E40, 46B20

\section{Introduction}

Let $\left(X,\|\cdot\|_{X}\right)$ be a real Banach space, and let $B(X)$ and $S(X)$ be the closed unit ball and the unit sphere of $X$, repsectively. By $X^{*}$ denote the dual space of $X$. In the sequel $\mathbb{N}$ and $\mathbb{R}$ denote the set of natural numbers and the set of reals, respectively.

Yunan Cui: Harbin Univ. of Sci. \& Techn., Dept. Math., Yuefu Road 52, 150080 Harbin, P.R. China; cuiya@mail.hrbust.edu.cn

H. Hudzik: Adam Mickiewicz Univ., Fac. Math. \& Comp. Sci., ul. Umultowska 87, PL - 61-614 Poznań, Poland; hudzik@amu.edu.pl

R. Płuciennik, Poznań Univ. Techn., Inst. Math., Piotrowo 3A, PL - 60-965 Poznań, Poland; rplucien@math.put.poznan.pl

ISSN 0232-2064 / \$2.50 C Heldermann Verlag Berlin 
Before starting with our results, we need to recall some notions.

A point $x \in S(X)$ is said to be an extreme point of $B(X)$ if $x$ cannot be written as the arithmetic mean $\frac{1}{2}(y+z)$ of two distinct points $y, z \in S(X)$. A Banach space $X$ is said to be rotund if every point in $S(X)$ is an extreme point. A point $x \in S(X)$ is said to be a strongly extreme point of $B(X)$ if for every sequences $\left(y_{n}\right),\left(z_{n}\right)$ in $X$ such that $\left\|y_{n}\right\|_{X},\left\|z_{n}\right\|_{X} \rightarrow 1$ the condition $y_{n}+z_{n}=2 x$ for any $n \in \mathbb{N}$ implies $\left\|y_{n}-x\right\|_{X} \rightarrow 0$. A Banach space $X$ is said to be midpoint locally uniformly rotund if every point of $S(X)$ is strongly extreme.

We denote by ext $B(X)$ and sext $B(X)$ the sets of extreme points and of strongly extreme points, respectively, of $B(X)$.

The notion of extreme point plays an important role in some branches of mathematics. For example, the Krein-Milman theorem, Choquet integral representation theorem, Rainwater theorem on convergence in the weak topology, Bessaga-Pełczyński theorem and Elton test for unconditional convergence are strongly connected with this notion. In [26], using the principle of local reflexivity, a remarkable theorem describing connections between extreme points of $S(X)$ and strongly extreme points of $S(X)$ is proved. Namely, a Banach space $X$ is midpoint locally uniformly rotund if and only if every point of $S(X)$ is an extreme point in $X^{* *}$. Another proof of this theorem based on Goldstein's theorem is given in [8]. Analyzing the proof of this fact one can easily see its local version, namely, if $x \in S(X)$ is a strongly extreme point in $X$, then $\kappa(x)$ is an extreme point in $X^{* *}$, where $\kappa$ is the mapping of canonical embedding of $X$ into $X^{* *}$.

The aim of this paper is to give criteria for extreme points and strongly extreme points of the unit ball of Orlicz spaces generated by arbitrary Orlicz functions (that is, Orlicz functions which vanish outside zero and which attain infinite values to the right of some point $u>0$ are not excluded) and equipped with the Orlicz norm. We prove that the necessary conditions for a point $x$ from the unit sphere to be an extreme point presented in [16] are also sufficient. We will give sufficient conditions under which $\operatorname{ext} B\left(L_{\Phi}^{0}\right)=\emptyset$. It is an important observation because such space lacks Krein-Milmann Property, so it is not isometric to any dual space. As we will see below, the fact that the degenerated Orlicz functions are not excluded in our considerations is of great interest. Namely, the classical spaces $L^{1}+L^{\infty}$ and $L^{1} \cap L^{\infty}$ which are important in the interpolation theory as well as the spaces $L^{p} \cap L^{\infty} \quad(1<p<$ $\infty)$ become a special cases of Orlicz spaces investigated in this paper.

A map $\Phi: \mathbb{R} \rightarrow[0, \infty]$ is said to be an Orlicz function if it is even, convex, left continuous on whole of $\mathbb{R}^{+}, \Phi(0)=0$ and $\Phi$ is not identically equal to zero. 
For any Orlicz function $\Phi$ we set

$$
\begin{aligned}
& a(\Phi)=\sup \{u \geq 0: \Phi(u)=0\} \\
& b(\Phi)=\sup \{u>0: \Phi(u)<\infty\} .
\end{aligned}
$$

We say an Orlicz function $\Phi$ satisfies the $\Delta_{2}$-condition for all $u \in \mathbb{R}$ (at infinity) [at zero] if there are positive constants $K$ and $u_{0}$ with $0<\Phi\left(u_{0}\right)<\infty$ such that $\Phi(2 u) \leq K \Phi(u)$ holds for all $u \in \mathbb{R}$ (for every $|u| \geq u_{0}$ ) [for every $\left.|u| \leq u_{0}\right]$. We denote these conditions by $\Phi \in \Delta_{2}\left(\Phi \in \Delta_{2}(\infty)\right)\left[\Phi \in \Delta_{2}(0)\right]$, respectively. Obviously, $\Phi \in \Delta_{2}$ if and only if $\Phi \in \Delta_{2}(\infty)$ and $\Phi \in \Delta_{2}(0)$.

Let $(T, \Sigma, \mu)$ be a measure space with a $\sigma$-finite, non-atomic and complete measure $\mu$ and $L^{0}(\mu)$ be the set of all $\mu$-equivalence classes of real and $\Sigma$ measurable functions defined on $T$. For a given Orlicz function $\Phi$ we define on $L^{0}(\mu)$ a convex functional (called a pseudomodular, see [22]) by

$$
I_{\Phi}(x)=\int_{T} \Phi(x(t)) d \mu .
$$

We define the Orlicz space $L_{\Phi}$ generated by an Orlicz function $\Phi$ by the formula

$$
L_{\Phi}=\left\{x \in L^{0}(\mu): I_{\Phi}(c x)<\infty \text { for some } c>0 \text { depending on } x\right\} .
$$

This space is usually equipped with the Luxemburg norm

$$
\|x\|_{\Phi}=\inf \left\{\varepsilon>0: I_{\Phi}\left(\frac{x}{\varepsilon}\right) \leq 1\right\}
$$

or with the equivalent one

$$
\|x\|_{\Phi}^{0}=\sup \left\{\int_{T}|x(t) y(t)| d \mu: y \in L^{\Psi}, I_{\Psi}(y) \leq 1\right\}
$$

called the Orlicz norm, where the function $\Psi$ is defined by the formula

$$
\Psi(u)=\sup \{|u| v-\Phi(v): v \geq 0\}
$$

and called complementary to $\Phi$ in the sense of Young. It is proved in [15] that for any Orlicz function $\Phi$ the Amemiya formula for the Orlicz norm

$$
\|x\|_{\Phi}^{0}=\inf _{k>0} \frac{1}{k}\left(1+I_{\Phi}(k x)\right)
$$

is true. The set of all $k>0$ at which the infimum in the Amemiya formula for $\|x\|_{\Phi}^{0}$ is attained (for fixed $x \in L_{\Phi}$ ) will be denoted by $K(x)$. In particular, the 
set $K(x)$ can be empty if the Orlicz space $L_{\Phi}$ is generated by an Orlicz function such that the function $R(u)=A u-\Phi(u)$ is bounded, where $A=\lim _{u \rightarrow \infty} \frac{\Phi(u)}{u}$ (see [3]). Moreover, for any $x \in L_{\Phi}$ define

$$
\theta(x)=\sup \left\{c>0: I_{\Phi}(c x)<\infty\right\}
$$

To simplify notations, we put $L_{\Phi}=\left(L_{\Phi},\|\cdot\|_{\Phi}\right)$ and $L_{\Phi}^{0}=\left(L_{\Phi},\|\cdot\|_{\Phi}^{0}\right)$.

We say $w$ is a point of strict convexity of $\Phi$ (we write $w \in S C(\Phi)$ ) if for every $u, v \in \mathbb{R}$ such that $u \neq v$ and $w=\frac{1}{2}(u+v)$ there holds

$$
\Phi\left(\frac{u+v}{2}\right)<\frac{1}{2}(\Phi(u)+\Phi(v))
$$

For more details on Orlicz spaces we refer to $[2,19,22,24]$.

\section{General results}

The following result proved in [4] for the Orlicz sequence space $l_{\Phi}^{0}$ is also true in the function space $L_{\Phi}^{0}$.

Proposition 1. Let $x \in L_{\Phi}^{0} \backslash\{0\}$. If $K(x)=\emptyset$, then

$$
\|x\|_{\Phi}^{0}=\lim _{k \rightarrow \theta(x)-} \frac{1}{k}\left(1+I_{\Phi}(k x)\right) .
$$

Proposition 1 leads to the following

\section{Corollary 1.}

(a) If $x \in L_{\Phi}^{0}$ and $\theta(x)<\infty$, then $K(x) \neq \emptyset$.

(b) If $K(x)=\emptyset$, then $\left\|x \chi_{B}\right\|_{\Phi}^{0}=A\left\|x \chi_{B}\right\|_{L^{1}}$ for any $B \in \Sigma$, where $A=\lim _{u \rightarrow \infty} \frac{\Phi(u)}{u}$.

Proof. Both statements (a) and (b) can be proved on the base of Proposition 1 by the same argumentation as in [4] and the fact that $K\left(x \xi_{B}\right)=\emptyset$ for any $B \in \Sigma$ whenever $K(x)=\emptyset$

Theorem 1. Let $\Phi$ be an arbitrary Orlicz function. Then $x \in S\left(L_{\Phi}^{0}\right)$ is an extreme point of the unit ball $B\left(L_{\Phi}^{0}\right)$ if and only if:

(a) the set $K(x)$ consists of one element from $(0,+\infty)$

(b) $k x(t) \in S C(\Phi)$ for $\mu$-a.e. $t \in T$, where $\{k\}=K(x)$.

Proof. Necessity. First, we will prove the necessity of $K(x) \neq \emptyset$. Although this fact was proved in [16], we will present here another very short proof. Suppose that $x$ is an extreme point of the unit ball $B\left(L_{\Phi}^{0}\right)$ and 
$K(x)=\emptyset$. We can find a number $a>0$ such that the set $\{t \in T:|x(t)| \geq a\}$ has positive and finite measure. We can assume without loss of generality that the measure of the set $T_{0}=\{t \in T: x(t) \geq a\}$ is also positive and finite. Take two subsets $T_{1}$ and $T_{2}$ of $T_{0}$ such that $\mu\left(T_{1}\right)=\mu\left(T_{2}\right)>0$. Choose $\varepsilon \in(0, a)$ and put

$$
\begin{aligned}
& x_{1}(t)=x(t) \chi_{T \backslash\left(T_{1} \cup T_{2}\right)}+(x(t)+\varepsilon) \chi_{T_{1}}+(x(t)-\varepsilon) \chi_{T_{2}} \\
& x_{2}(t)=x(t) \chi_{T \backslash\left(T_{1} \cup T_{2}\right)}+(x(t)-\varepsilon) \chi_{T_{1}}+(x(t)+\varepsilon) \chi_{T_{2}} .
\end{aligned}
$$

Obviously, $x=\frac{1}{2}\left(x_{1}+x_{2}\right)$ and $x_{1} \neq x_{2}$. Moreover, by Corollary $1 /(\mathrm{b})$ we have

$$
\left\|x_{i}\right\|_{\Phi}^{0} \leq A \int_{T}\left|x_{i}(t)\right| d \mu=A \int_{T}|x(t)| d \mu=\|x\|_{\Phi}^{0}=1
$$

for $i=1,2$. Hence $x_{1}, x_{2} \in B\left(L_{\Phi}^{0}\right)$. Therefore $x$ cannot be an extreme point of $B\left(L_{\Phi}^{0}\right)$. If $K(x) \neq \emptyset$ and $x \in S\left(L_{\Phi}^{0}\right)$ is an extreme point, then $k x(t)$ must be points of strict convexity of $\Phi$ for $\mu$-a.e. $t \in T$ and $K(x)$ must be a singleton (because otherwise $x$ is not an extreme point, see [16]).

Sufficiency. We first prove that for $x_{1}, x_{2} \in S\left(L_{\Phi}^{0}\right)$ with $x=\frac{x_{1}+x_{2}}{2}$ at least one of the sets $K\left(x_{1}\right)$ or $K\left(x_{2}\right)$ is non-empty. Suppose that $K\left(x_{1}\right)=\emptyset$ and $K\left(x_{2}\right)=\emptyset$. Then

$$
\begin{aligned}
2 & =\left\|x_{1}+x_{2}\right\|_{\Phi}^{0} \\
& <A \int_{T}\left|x_{1}(t)+x_{2}(t)\right| d \mu \\
& \leq A \int_{T}\left|x_{1}(t)\right| d \mu+A \int_{T}\left|x_{2}(t)\right| d \mu \\
& =\left\|x_{1}\right\|_{\Phi}^{0}+\left\|x_{2}\right\|_{\Phi}^{0} \\
& =2 .
\end{aligned}
$$

The first sharp inequality follows from the fact that $+\infty \notin K(x)$ because $K(x)=\{k\}$, where $0<k<+\infty$. This contradiction shows that $K\left(x_{1}\right) \neq \emptyset$ or $K\left(x_{2}\right) \neq \emptyset$.

Now we will prove that $K\left(x_{1}\right) \neq \emptyset$ and $K\left(x_{2}\right) \neq \emptyset$. Otherwise, we can assume without loss of generality that $K\left(x_{1}\right) \neq \emptyset$ and $K\left(x_{2}\right)=\emptyset$. Put

$$
\begin{aligned}
& {\left[x_{1}, x\right)=\left\{(1-\lambda) x_{1}+\lambda x: 0 \leq \lambda<1\right\}} \\
& \left(x, x_{2}\right]=\left\{(1-\lambda) x+\lambda x_{2}: 0<\lambda \leq 1\right\} .
\end{aligned}
$$

Next we will prove that $K(y) \neq \emptyset$ for all $y \in\left[x_{1}, x\right)$ and $K(y)=\emptyset$ for all $y \in\left(x, x_{2}\right]$. Assume first for the contrary that there is $x_{3} \in\left[x_{1}, x\right)$ such that 
$K\left(x_{3}\right)=\emptyset$. Then there exists $\lambda_{3} \in[0,1)$ such that $x_{3}=\left(1-\lambda_{3}\right) x_{1}+\lambda_{3} x$.

Since $x_{1}=2 x-x_{2}$, we have

$$
x_{3}=\left(1-\lambda_{3}\right)\left(2 x-x_{2}\right)+\lambda_{3} x=\left(2-\lambda_{3}\right) x-\left(1-\lambda_{3}\right) x_{2} .
$$

Hence $x=\frac{1}{2-\lambda_{3}} x_{3}+\frac{1-\lambda_{3}}{2-\lambda_{3}} x_{2}$. Therefore

$$
\begin{aligned}
1 & =\|x\|_{\Phi}^{0} \\
& <A \int_{T}\left|\frac{1}{2-\lambda_{3}} x_{3}(t)+\frac{1-\lambda_{3}}{2-\lambda_{3}} x_{2}(t)\right| d \mu \\
& \leq \frac{A}{2-\lambda_{3}} \int_{T}\left|x_{3}(t)\right| d \mu+\frac{1-\lambda_{3}}{2-\lambda_{3}} A \int_{T}\left|x_{2}(t)\right| d \mu \\
& =\frac{1}{2-\lambda_{3}}\left\|x_{3}\right\|_{\Phi}^{0}+\frac{1-\lambda_{3}}{2-\lambda_{3}}\left\|x_{2}\right\|_{\Phi}^{0} \\
& =1,
\end{aligned}
$$

a contradiction.

Assume now for the contrary that there is $x_{4} \in\left(x, x_{2}\right]$ such that $K\left(x_{4}\right) \neq$ $\emptyset$. We can find $x_{5} \in\left[x_{1}, x\right)$ such that $x=\frac{x_{4}+x_{5}}{2}$ and $x_{4} \neq x_{5}$. Therefore, there are $k_{4} \geq 1$ and $k_{5} \geq 1$ such that

$$
\begin{aligned}
\left\|x_{4}\right\|_{\Phi}^{0} & =\frac{1}{k_{4}}\left(1+I_{\Phi}\left(k_{4} x_{4}\right)\right) \\
\left\|x_{5}\right\|_{\Phi}^{0} & =\frac{1}{k_{5}}\left(1+I_{\Phi}\left(k_{5} x_{5}\right)\right) .
\end{aligned}
$$

By the convexity of the modular $I_{\Phi}$ we have

$$
\begin{aligned}
I_{\Phi}\left(\frac{k_{4} k_{5}}{k_{4}+k_{5}} 2 x\right) & =I_{\Phi}\left(\frac{k_{4} k_{5}}{k_{4}+k_{5}}\left(x_{4}+x_{5}\right)\right) \\
& =I_{\Phi}\left(\frac{k_{5}}{k_{4}+k_{5}} k_{4} x_{4}+\frac{k_{4}}{k_{4}+k_{5}} k_{5} x_{5}\right) \\
& \leq \frac{k_{5}}{k_{4}+k_{5}} I_{\Phi}\left(k_{4} x_{4}\right)+\frac{k_{4}}{k_{4}+k_{5}} I_{\Phi}\left(k_{5} x_{5}\right) .
\end{aligned}
$$

Hence

$$
\begin{aligned}
2 & =2\|x\|_{\Phi}^{0} \\
& \leq \frac{k_{4}+k_{5}}{k_{4} k_{5}}\left(1+I_{\Phi}\left(\frac{k_{4} k_{5}}{k_{4}+k_{5}} 2 x\right)\right) \\
& \leq \frac{k_{4}+k_{5}}{k_{4} k_{5}}\left(1+\frac{k_{5}}{k_{4}+k_{5}} I_{\Phi}\left(k_{4} x_{4}\right)+\frac{k_{4}}{k_{4}+k_{5}} I_{\Phi}\left(k_{5} x_{5}\right)\right) \\
& \leq \frac{1}{k_{4}}\left(1+I_{\Phi}\left(k_{4} x_{4}\right)\right)+\frac{1}{k_{5}}\left(1+I_{\Phi}\left(k_{5} x_{5}\right)\right) \\
& =2 .
\end{aligned}
$$


Consequently, all inequalities from the last three lines are equalities in fact. Therefore $\frac{2 k_{4} k_{5}}{k_{4}+k_{5}}=k$ and

$$
\Phi(k x(t))=\frac{k_{5}}{k_{4}+k_{5}} \Phi\left(k_{4} x_{4}(t)\right)+\frac{k_{4}}{k_{4}+k_{5}} \Phi\left(k_{5} x_{5}(t)\right)
$$

for $\mu$-a.e. $t \in T$. By the assumption that $\Phi$ is strictly convex at $k x(t)$ for $\mu$-a.e. $t \in T$, it follows that $k_{4} x_{4}(t)=k_{5} x_{5}(t)=k x(t)$ for $\mu$-a.e. $t \in T$. Since $x_{4}, x_{5}, x \in S\left(L_{\Phi}^{0}\right)$, we get $k_{4}=k_{5}=k$, which gives $x_{4}=x_{5}=x$. This contradicts the inequality $x_{4} \neq x_{5}$. Thus $K(y)=\emptyset$ for any $y \in\left(x, x_{2}\right]$. Take $x_{n}=\left(1-\frac{1}{n}\right) x+\frac{1}{n} x_{2}$ for all $n \in \mathbb{N}$. Then $x_{n} \in\left(x, x_{2}\right]$ for all $n \in \mathbb{N}$. Hence $K\left(x_{n}\right)=\emptyset$ and consequently $\left\|x_{n}\right\|_{\Phi}^{0}=A \int_{T}\left|x_{n}(t)\right| d \mu$ for all $n \in \mathbb{N}$. Note that $x_{n} \rightarrow x$ as $n \rightarrow \infty$ with respect to the norm $\|\cdot\|_{\Phi}^{0}$ and $\lim _{n \rightarrow \infty}\left|x_{n}(t)\right|=|x(t)|$ for $\mu$-a.e. $t \in T$. Since $K(x)=\{k\}$, with $0<k<+\infty$, we have

$$
\|x\|_{\Phi}^{0}=\lim _{n \rightarrow \infty}\left\|x_{n}\right\|_{\Phi}^{0}=\lim _{n \rightarrow \infty} A \int_{T}\left|x_{n}(t)\right| d \mu \geq A \int_{T}|x(t)| d \mu>\|x\|_{\Phi}^{0},
$$

a contradiction. Therefore $K\left(x_{1}\right) \neq \emptyset$ and $K\left(x_{2}\right) \neq \emptyset$. Now, repeating the same procedure as above, putting $x_{1}$ and $x_{2}$ instead of $x_{4}$ and $x_{5}$, respectively, we get

$$
k_{1} x_{1}(t)=k_{2} x_{2}(t)=k x(t)
$$

for $\mu$-a.e. $t \in T$. Hence, by the fact that $x_{1}, x_{2}, x \in S\left(L_{\Phi}^{0}\right)$, we have $k_{1}=k_{2}=$ $k$ and consequently, $x_{1}=x_{2}=x$. Thus $x$ is an extreme point of $B\left(L_{\Phi}^{0}\right)$

Corollary 2. The Orlicz space $L_{\Phi}^{0}$ is rotund if and only if:

(a) $\Phi$ is strictly convex

(b) $\lim _{u \rightarrow \infty} R(u)=\infty$ where $R(u)=A|u|-\Phi(u)$ with $A=\lim _{u \rightarrow \infty} \frac{\Phi(u)}{u}$.

Proof. Sufficiency. Taking any $x \in S\left(L_{\Phi}^{0}\right)$, we need to prove that $x$ is an extreme point of $S\left(L_{\Phi}^{0}\right)$. Condition (b) guarantees that $K(x) \neq \emptyset$ (see $[3])$. By condition $(\mathrm{a}), k x(t) \in S C(\Phi)$ for $\mu$-a.e. $t \in T$. Therefore, in view of Theorem $1, x$ is an extreme point of $B\left(L_{\Phi}^{0}\right)$.

Necessity. Let us first prove the necessity of condition (b). Assume that this condition is not satisfied. Then there is $x \in S\left(L_{\Phi}^{0}\right)$ such that $K(x)=\emptyset$ (see [3]). Consequently, if $B \in \Sigma \cap \operatorname{supp}(x)$ and $0<\mu(B)<\mu(\operatorname{supp}(x))$, then $K\left(x \chi_{B}\right)=\emptyset$ and $K\left(x \chi_{B^{\prime}}\right)=\emptyset$, where $B^{\prime}=\operatorname{supp}(x) \backslash B$. This yields that $A<\infty$ and, by Corollary $1 /(\mathrm{b}),\|x\|_{\Phi}^{0}=A\|x\|_{L^{1}},\left\|x \chi_{B}\right\|_{\Phi}^{0}=A\left\|x \chi_{B}\right\|_{L^{1}}$ and $\left\|x \chi_{B^{\prime}}\right\|_{\Phi}^{0}=A\left\|x \chi_{B^{\prime}}\right\|_{L^{1}}$. Therefore,

$$
\|x\|_{\Phi}^{0}=\left\|x \chi_{B}\right\|_{\Phi}^{0}+\left\|x \chi_{B^{\prime}}\right\|_{\Phi}^{0}
$$


For $\alpha=\left\|x \chi_{B}\right\|_{\Phi}^{0}$ and $\beta=\left\|x \chi_{B^{\prime}}\right\|_{\Phi}^{0}$, we have $\alpha, \beta \in(0,1)$ with $\alpha+\beta=1$. Moreover, defining $y=\frac{x \chi_{B}}{\left\|x \chi_{B}\right\|_{\Phi}^{0}}$ and $z=\frac{x \chi_{B^{\prime}}}{\left\|x \chi_{B^{\prime}}\right\|_{\Phi}^{0}}$, we have $y, z \in S\left(L_{\Phi}^{0}\right)$ and

$$
\alpha y+\beta z=x \chi_{B}+x \chi_{B^{\prime}}=x .
$$

This means that $x$ is not an extreme point of $B\left(L_{\Phi}^{0}\right)$ and so $L_{\Phi}^{0}$ is not rotund.

Using now condition (b), the necessity of which has already been proved, we have that $K(x) \neq \emptyset$ for any $x \in L_{\Phi}^{0} \backslash\{0\}$ (see [3]). Consequently, the necessity of condition (a) follows from Theorem 1 $\emptyset$.

The next corollary gives sufficient conditions under which the ext $B\left(L_{\Phi}^{0}\right)=$

Corollary 3. If one of the conditions

(i) $I_{\Psi}\left(p \circ u_{0} \chi_{T}\right)<1$ where $u_{0}=\sup \{u \geq a(\Phi): u \in S C(\Phi)\}$

(ii) $S C(\Phi) \backslash\{0\}=\emptyset$

is satisfied, then $\operatorname{ext} B\left(L_{\Phi}^{0}\right)=\emptyset$.

Proof. Suppose ext $B\left(L_{\Phi}^{0}\right) \neq \emptyset$. Then, by Theorem 1, there are $x_{0} \in$ $S\left(L_{\Phi}^{0}\right)$ and exactly one $k_{0} \geq 1$ such that

$$
\left\|x_{0}\right\|_{\Phi}^{0}=\frac{1}{k_{0}}\left(1+I_{\Phi}\left(k_{0} x_{0}\right)\right)
$$

and $k_{0} x_{0}(t) \in S C(\Phi)$ for $\mu$-a.e. $t \in T$. It is well known that $K\left(x_{0}\right)=\left[k^{*}, k^{* *}\right]$ where

$$
\begin{gathered}
k^{*}=k^{*}\left(x_{0}\right)=\inf \left\{k>0: I_{\Psi}\left(p \circ k\left|x_{0}\right|\right) \geq 1\right\} \\
k^{* *}=k^{* *}\left(x_{0}\right)=\sup \left\{k>0: I_{\Psi}\left(p \circ k\left|x_{0}\right|\right) \leq 1\right\} .
\end{gathered}
$$

Since $K\left(x_{0}\right)$ is a singleton, $k_{0}=k^{*}=k^{* *}$. If condition (i) is satisfied, then $k_{0}\left|x_{0}(t)\right| \leq u_{0}$ for $\mu$ a.e. $t \in T$. Hence

$$
I_{\Psi}\left(p \circ k\left|x_{0}\right|\right) \leq I_{\Psi}\left(p \circ u_{0} \chi_{T}\right)<1
$$

and consequently the set

$$
\left\{k>0: I_{\Psi}\left(p \circ k\left|x_{0}\right|\right) \geq 1\right\}
$$

is empty, i.e. $K\left(x_{0}\right)=\emptyset$. Hence, by Theorem 1, $x_{0}$ cannot be an extreme point of $B\left(L_{\Phi}^{0}\right)$, a contradiction.

If condition (ii) is satisfied, then, by Theorem $1, k_{0} x_{0}(t)=0$ for $\mu$-a.e. $t \in T$ and, consequently, $x_{0}(t)=0$ for $\mu$-a.e. $t \in T$. Therefore, $x_{0} \notin S\left(L_{\Phi}^{0}\right)$, a contradiction. This shows that in this case $\operatorname{ext} B\left(L_{\Phi}^{0}\right)=\emptyset$, which finishes the proof 
Remark 1. The condition $\lim _{u \rightarrow \infty} R(u)=\infty$ is equivalent to the fact that $\lim _{u \rightarrow A} \Psi(u)=\infty$, where $R=A|u|-\Phi(u)$ and the constant $A$ are defined as above and $\Psi$ is the function complementary to $\Phi$ in the sense of Young.

Theorem 2. Assume $\Phi$ is an Orlicz function and $x \in S\left(L_{\Phi}^{0}\right)$. Then $x$ is a strongly extreme point of $B\left(L_{\Phi}^{0}\right)$ if and only if the following conditions are satisfied:

(a) The set $K(x)$ is a singleton, that is $K(x)=\{k\}$, where $k>0$.

(b) $k x(t) \in S C(\Phi)$ for $\mu$-a.e. $t \in T$.

(c) Either $\Phi(b(\Phi))<\infty$ and $x$ is of the form $k|x(t)|=b(\Phi)$ for $\mu$-a.e. $t \in T$ or $\Phi \in \Delta_{2}(\infty)$ and at least one of the conditions

(i) $\mu(T)<\infty$

(ii) $a(\Phi)>0$

(iii) $\Phi \in \Delta_{2}(0)$

is satisfied.

Proof. Necessity. Let $x$ be a strongly extreme point of $B\left(L_{\Phi}^{0}\right)$. Since strongly extreme points are extreme points, so the necessity of conditions (a) and (b) follows from Theorem 1. Consequently, we need only to prove the necessity of condition (c). For, assuming that $b(\Phi)=\infty$, we first prove the necessity of $\Phi \in \Delta_{2}(\infty)$. Assume for the contrary that $\Phi \notin \Delta_{2}(\infty)$. Then there is a sequence $\left(u_{n}\right)$ of positive numbers such that $u_{n} \nearrow \infty$ and $\Phi\left(2 u_{n}\right)>2^{n} \Phi\left(u_{n}\right)$ for every $n \in \mathbb{N}$. Take a number $a>0$ such that the set $T_{0}=\{t \in T:|x(t)| \leq a\}$ has a positive measure. Next, passing to a subsequence of $\left(u_{n}\right)$ if necessary, we may assume that for any $n \in \mathbb{N}$ there is $T_{n} \in \Sigma$ with $T_{n} \subset T_{0}$ such that $\Phi\left(u_{n}\right) \mu\left(T_{n}\right)=\frac{1}{2^{n}}$. Define

$$
\begin{aligned}
& x_{n}(t)=x(t) \chi_{T \backslash T_{n}}(t)+\left(x(t)+\frac{u_{n}}{k} \operatorname{sign} x(t)\right) \chi_{T_{n}}(t) \\
& y_{n}(t)=x(t) \chi_{T \backslash T_{n}}(t)+\left(x(t)-\frac{u_{n}}{k} \operatorname{sign} x(t)\right) \chi_{T_{n}}(t) \\
& z_{n}(t)=x(t) \chi_{T \backslash T_{n}}(t)+\frac{u_{n}}{k} \operatorname{sign} x(t) \chi_{T_{n}}(t)
\end{aligned}
$$

for every $n \in \mathbb{N}$. Then $x_{n}+y_{n}=2 x$ for any $n \in \mathbb{N}$ and, by $\mu\left(T_{n}\right) \rightarrow 0$ as $n \rightarrow \infty$, we have

$$
\lim _{n \rightarrow \infty}\left\|x_{n}-z_{n}\right\|_{\Phi}^{0}=\lim _{n \rightarrow \infty}\left\|x \chi_{T_{n}}\right\|_{\Phi}^{0} \leq a \lim _{n \rightarrow \infty}\left\|\chi_{T_{n}}\right\|_{\Phi}^{0}=0 .
$$

Since $\left|y_{n}\right| \leq\left|x_{n}\right|$ for any $n \in \mathbb{N}$ we have

$$
\begin{aligned}
\limsup _{n \rightarrow \infty}\left\|y_{n}\right\|_{\Phi}^{0} & \leq \limsup _{n \rightarrow \infty}\left\|x_{n}\right\|_{\Phi}^{0} \\
& =\limsup _{n \rightarrow \infty}\left\|z_{n}\right\|_{\Phi}^{0}
\end{aligned}
$$




$$
\begin{aligned}
& \leq \limsup _{n \rightarrow \infty} \frac{1}{k}\left[1+I_{\Phi}\left(k x \chi_{T \backslash T_{n}}\right)+\int_{T_{n}} \Phi\left(u_{n}\right) d \mu\right] \\
& \leq \limsup _{n \rightarrow \infty}\left[\frac{1}{k}\left(1+I_{\Phi}(k x)\right)+\frac{1}{k} \Phi\left(u_{n}\right) \mu\left(T_{n}\right)\right] \\
& =1+\frac{1}{k} \limsup _{n \rightarrow \infty} \Phi\left(u_{n}\right) \mu\left(T_{n}\right) \\
& =1 .
\end{aligned}
$$

Moreover,

$$
0=\lim _{n \rightarrow \infty}\left\|x \chi_{T_{n}}\right\|_{\Phi}^{0}=\lim _{n \rightarrow \infty}\left\|x-x \chi_{T \backslash T_{n}}\right\|_{\Phi}^{0}
$$

whence $\lim _{n \rightarrow \infty}\left\|x \chi_{T \backslash T_{n}}\right\|_{\Phi}^{0}=\|x\|_{\Phi}^{0}=1$. Hence, by $\left\|x_{n}\right\|_{\Phi}^{0} \geq\left\|y_{n}\right\|_{\Phi}^{0} \geq$ $\left\|x \chi_{T \backslash T_{n}}\right\|_{\Phi}^{0}$ for every $n \in \mathbb{N}$, we have

$$
\liminf _{n \rightarrow \infty}\left\|x_{n}\right\|_{\Phi}^{0} \geq \liminf _{n \rightarrow \infty}\left\|y_{n}\right\|_{\Phi}^{0} \geq \liminf _{n \rightarrow \infty}\left\|x \chi_{T \backslash T_{n}}\right\|_{\Phi}^{0}=1 .
$$

The last inequalities and inequalities (1) yield

$$
\lim _{n \rightarrow \infty}\left\|x_{n}\right\|_{\Phi}^{0}=\lim _{n \rightarrow \infty}\left\|y_{n}\right\|_{\Phi}^{0}=1=\|x\|_{\Phi}^{0} .
$$

However, we have for any $n \in \mathbb{N}$,

$$
I_{\Phi}\left(2 k\left(x_{n}-x\right)\right)=\Phi\left(2 u_{n}\right) \mu\left(T_{n}\right)>2^{n} \Phi\left(u_{n}\right) \mu\left(T_{n}\right)=1
$$

whence $\left\|x_{n}-x\right\|_{\Phi}^{0} \geq\left\|x_{n}-x\right\|_{\Phi} \geq 2 k$, which contradicts the fact that $x$ is a strongly extreme point.

Now we will prove that if $\mu(T)=\infty, a(\Phi)=0, b(\Phi)=\infty$ and $\Phi \in \Delta_{2}(\infty)$, then $\Phi \in \Delta_{2}(0)$, whenever $x$ is a strongly extreme point of $B\left(L_{\Phi}^{0}\right)$. First we will show that there exists a set $A \in \Sigma$ such that $I_{\Phi}\left(2 x \chi_{A}\right)<\infty$ and $\mu(A)=$ $\infty$. Let $\left(u_{n}\right)$ be a sequence of positive numbers such that $\Phi\left(2 u_{n}\right)<\frac{1}{2^{n+1}}$ and define

$$
K_{n}=\sup \left\{\frac{\Phi(2 u)}{\Phi(u)}: u \geq u_{n}\right\} .
$$

Then $\Phi(2 u) \leq K_{n} \Phi(u)$ for all $u \geq u_{n}(n \in \mathbb{N})$. By the assumptions that the measure $\mu$ is non-atomic and $\mu(T)=\infty$ one can find a sequence $\left(A_{n}\right)$ in $\Sigma$ such that $\mu\left(A_{n}\right)=1$ for any $n \in \mathbb{N}$ and $\mu\left(A_{m} \cap A_{n}\right)=0$ for any $m, n \in \mathbb{N}$ with $m \neq n$. Since $I_{\Phi}(k x)<\infty$, we get $I_{\Phi}\left(k x \chi_{A_{n}}\right) \rightarrow 0$ as $n \rightarrow \infty$. Consequently, there exists a subsequence $\left(n_{j}\right)$ of natural numbers such that $I_{\Phi}\left(k x \chi_{A_{n_{j}}}\right)<\frac{1}{2^{j+1} K_{j}}$ for all $j \in \mathbb{N}$. Define

$$
A_{n_{j}}^{1}=\left\{t \in A_{n_{j}}: k|x(t)| \geq u_{j}\right\}, \quad A_{n_{j}}^{2}=A_{n_{j}} \backslash A_{n_{j}}^{1}, \quad A=\cup_{j=1}^{\infty} A_{n_{j}} .
$$


Then

$$
\begin{aligned}
I_{\Phi}\left(2 k x \chi_{A}\right) & =\sum_{j=1}^{\infty} I_{\Phi}\left(2 k x \chi_{A_{n_{j}}}\right) \\
& =\sum_{j=1}^{\infty} I_{\Phi}\left(2 k x \chi_{A_{n_{j}}^{1}}\right)+\sum_{j=1}^{\infty} I_{\Phi}\left(2 k x \chi_{A_{n_{j}}^{2}}\right) \\
& \leq \sum_{j=1}^{\infty} K_{j} I_{\Phi}\left(k x \chi_{A_{n_{j}}^{1}}\right)+\sum_{j=1}^{\infty} \Phi\left(2 u_{j}\right) \mu\left(A_{n_{j}}^{2}\right) \\
& <\sum_{j=1}^{\infty} \frac{1}{2^{(j+1)}}+\sum_{j=1}^{\infty} \frac{1}{2^{(j+1)}} \\
& =1
\end{aligned}
$$

which means that $A$ is the desired set.

Now, we will show that there is a sequence $\left(B_{n}\right)$ of measurable subsets of $A$ such that $\mu\left(B_{n}\right)=\infty$ for all $n \in \mathbb{N}$ and $I_{\Phi}\left(2 k x \chi_{B_{n}}\right) \rightarrow 0$ as $n \rightarrow \infty$. For, define

$$
C_{n}=\left\{t \in A: \frac{1}{n}<|x(t)| \leq n\right\}
$$

Then $\cup_{n=1}^{\infty} C_{n}=A$ up to a set of measure zero and $I_{\Phi}\left(2 k x \chi_{C_{n}}\right) \rightarrow I_{\Phi}\left(2 k x \chi_{A}\right)$ by the Beppo-Levi theorem, whence $I_{\Phi}\left(2 k x \chi_{A \backslash C_{n}}\right) \rightarrow 0$ as $n \rightarrow \infty$. By $\mu\left(C_{n}\right)<\infty$ for any $n \in \mathbb{N}$ we have $\mu\left(A \backslash C_{n}\right)=\infty$ for any $n \in \mathbb{N}$. Therefore, setting $B_{n}=A \backslash C_{n}$ for all $n \in \mathbb{N}$, we get the desired sequence.

Assume now that

$$
\mu(T)=\infty, \quad a(\Phi)=0, \quad b(\Phi)=\infty, \quad \Phi \in \Delta_{2}(\infty), \quad \Phi \notin \Delta_{2}(0)
$$

Then there exists a sequence $\left(u_{n}\right)$ of positive numbers such that $u_{n} \searrow 0$ as $n \rightarrow \infty$ and $\Phi\left(2 u_{n}\right)>2^{n} \Phi\left(u_{n}\right)$ for every $n \in \mathbb{N}$. For any $n \in \mathbb{N}$ choose $T_{n} \subset B_{n}$ with $T_{n} \in \Sigma$ such that $\Phi\left(u_{n}\right) \mu\left(T_{n}\right)=2^{-n}$ and define

$$
\begin{aligned}
& x_{n}=x+\frac{u_{n}}{2 k} \chi_{T_{n}} \operatorname{sign} x \\
& y_{n}=x-\frac{u_{n}}{2 k} \chi_{T_{n}} \operatorname{sign} x .
\end{aligned}
$$

Then $x_{n}+y_{n}=2 x$ for every $n \in \mathbb{N}$. Moreover, $|x| \leq\left|x_{n}\right|$ for any $n \in \mathbb{N}$, whence $\liminf \inf _{n \rightarrow \infty}\left\|x_{n}\right\|_{\Phi}^{0} \geq\|x\|_{\Phi}^{0}=1$. On the other hand, we have for each 
$n \in \mathbb{N}$

$$
\begin{aligned}
\left\|x_{n}\right\|_{\Phi}^{0} & \leq \frac{1}{k}\left(1+I_{\Phi}\left(k x_{n}\right)\right) \\
& =\frac{1}{k}\left(1+I_{\Phi}\left(k x \chi_{T \backslash T_{n}}\right)\right)+\int_{T_{n}} \Phi\left(\frac{2 k x(t)+u_{n}}{2}\right) d \mu \\
& \leq 1+\frac{1}{2}\left\{I_{\Phi}\left(2 k x \chi_{T_{n}}\right)+\Phi\left(u_{n}\right) \mu\left(T_{n}\right)\right\} \\
& \rightarrow 1 \quad(n \rightarrow \infty) .
\end{aligned}
$$

Consequently, $\lim \sup _{n \rightarrow \infty}\left\|x_{n}\right\|_{\Phi}^{0} \leq 1$, whence $\lim _{n \rightarrow \infty}\left\|x_{n}\right\|_{\Phi}^{0}=1$. Since $\left|y_{n}\right| \leq|x|$ for all $n \in \mathbb{N}$, we get $\lim \sup _{n \rightarrow \infty}\left\|y_{n}\right\|_{\Phi}^{0} \leq\|x\|_{\Phi}^{0}=1$.

In order to prove that $\lim _{n \rightarrow \infty}\left\|y_{n}\right\|_{\Phi}^{0}=1$, we need only to show that $\liminf _{n \rightarrow \infty}\left\|y_{n}\right\|_{\Phi}^{0} \geq 1$. Assume for the contrary that $\liminf _{n \rightarrow \infty}\left\|y_{n}\right\|_{\Phi}^{0}<1$. Then we get

$$
\begin{aligned}
2 & =\|2 x\|_{\Phi}^{0} \\
& =\lim _{n \rightarrow \infty}\left\|x_{n}+y_{n}\right\|_{\Phi}^{0} \\
& =\liminf _{n \rightarrow \infty}\left\|x_{n}+y_{n}\right\|_{\Phi}^{0} \\
& \left.\leq \liminf _{n \rightarrow \infty}\left\|x_{n}\right\|_{\Phi}^{0}+\left\|y_{n}\right\|_{\Phi}^{0}\right) \\
& <1+\liminf _{n \rightarrow \infty}\left\|y_{n}\right\|_{\Phi}^{0} \\
& <2
\end{aligned}
$$

- a contradiction. Therefore, $\lim _{n \rightarrow \infty}\left\|y_{n}\right\|_{\Phi}^{0}=1$. Moreover,

$$
I_{\Phi}\left(4 k\left(x_{n}-x\right)\right)=I_{\Phi}\left(2 u_{n} \chi_{T_{n}}\right)=\Phi\left(2 u_{n}\right) \mu\left(T_{n}\right)>2^{n} \Phi\left(u_{n}\right) \mu\left(T_{n}\right)=1
$$

for all $n \in \mathbb{N}$. Hence, by the definition of the Luxemburg norm, we have

$$
\left\|x_{n}-x\right\|_{\Phi}^{0} \geq\left\|x_{n}-x\right\|_{\Phi}>\frac{1}{4 k}
$$

for each $n \in \mathbb{N}$. Thus $x$ is not a strongly extreme point.

To finish the proof of the necessity we need to consider the case $b(\Phi)<$ $\infty$. First assume that $\lim _{u \rightarrow b(\Phi)_{-}} \Phi(u)=\infty$. Hence and by the inequality $I_{\Phi}(k x)<\infty$, we get $k|x(t)|<b(\Phi)$ for $\mu$-a.e. $t \in T$. Therefore, defining

$$
A_{n}=\left\{t \in T: k|x(t)|<\left(1-\frac{1}{n}\right) b(\Phi)\right\} \quad(n \in \mathbb{N})
$$

we have $A_{1} \subset A_{2} \subset \ldots$ and $\mu\left(T \backslash \cup_{n=1}^{\infty} A_{n}\right)=0$. Consequently, there is $m \in \mathbb{N}$ such that $\mu\left(A_{m}\right)>0$. Denote $A_{m}=A$ and $\lambda=\sqrt{1-\frac{1}{m}}$. Then 
$\frac{k|x(t)|}{\lambda} \leq \lambda b(\Phi)$ for every $t \in A$. Choose a sequence $\left(B_{n}\right)$ of measurable subsets of $A$ such that $\mu\left(B_{n}\right) \rightarrow 0$ as $n \rightarrow \infty$ and define

$$
\begin{aligned}
& x_{n}=x+\frac{(1-\lambda) b(\Phi)}{2 k} \operatorname{sign} x \chi_{B_{n}} \\
& y_{n}=x-\frac{(1-\lambda) b(\Phi)}{2 k} \operatorname{sign} x \chi_{B_{n}} .
\end{aligned}
$$

Then $|x| \leq\left|x_{n}\right|$ for all $n \in \mathbb{N}$, whence $\liminf _{n \rightarrow \infty}\left\|x_{n}\right\|_{\Phi}^{0} \geq\|x\|_{\Phi}^{0}=1$. Moreover,

$$
\begin{aligned}
\left\|x_{n}\right\|_{\Phi}^{0} & \leq \frac{1}{k}\left(1+I_{\Phi}\left(k x_{n}\right)\right) \\
& =\frac{1}{k}\left(1+I_{\Phi}\left(k x \chi_{T \backslash B_{n}}\right)\right)+\frac{1}{k} I_{\Phi}\left(\lambda \frac{k x}{\lambda} \chi_{B_{n}}+(1-\lambda) \frac{b(\Phi)}{2} \chi_{B_{n}}\right) \\
& \leq \frac{1}{k}\left(1+I_{\Phi}(k x)\right)+\lambda I_{\Phi}\left(\frac{k x}{\lambda} \chi_{B_{n}}\right)+(1-\lambda) \Phi\left(\frac{1}{2} b(\Phi)\right) \mu\left(B_{n}\right) \\
& \rightarrow \frac{1}{k}\left(1+I_{\Phi}(k x)\right) \\
& =\|x\|_{\Phi}^{0} \\
& =1 .
\end{aligned}
$$

Consequently, $\liminf _{n \rightarrow \infty}\left\|x_{n}\right\|_{\Phi}^{0} \leq 1$, whence $\lim _{n \rightarrow \infty}\left\|x_{n}\right\|_{\Phi}^{0}=1$. Since $\left|y_{n}\right| \leq|x|$ for all $n \in \mathbb{N}$, we get

$$
\limsup _{n \rightarrow \infty}\left\|y_{n}\right\|_{\Phi}^{0} \leq\|x\|_{\Phi}^{0}=1
$$

It is easy to prove that $\lim _{n \rightarrow \infty}\left\|y_{n}\right\|_{\Phi}^{0}=1$.

However, we have for any $n \in \mathbb{N}$

$$
I_{\Phi}\left(\frac{2 k}{1-\lambda}\left(x_{n}-x\right)\right)=\Phi(b(\Phi)) \mu\left(B_{n}\right)=\infty
$$

whence

$$
\left\|x_{n}-x\right\|_{\Phi}^{0} \geq\left\|x_{n}-x\right\|_{\Phi} \geq \frac{1-\lambda}{2 k} .
$$

Since $x_{n}+y_{n}=2 x$ for any $n \in \mathbb{N}$, this means that $x$ is not a strongly extreme point if $b(\Phi)<\infty$ and $\lim _{u \rightarrow b(\Phi)_{-}} \Phi(u)=\infty$.

Similarly we can prove that if $\Phi(b(\Phi))<\infty, x \in S\left(L_{\Phi}^{0}\right)$ and $k|x(t)|<b(\Phi)$ for $t \in A$, where $\mu(A)>0$, then $x$ is not a strongly extreme point. Therefore, if $b(\Phi)<\infty$ and $x \in S\left(L_{\Phi}^{0}\right)$ is a strongly extreme point, then it must be $\Phi(b(\Phi))<\infty$ and $k|x(t)|=b(\Phi)$ for $\mu$-a.e. $t \in T$. This finishes the proof of the necessity. 
Sufficiency. Suppose $x \in S\left(L_{\Phi}^{0}\right)$. By Theorem 1 and conditions (a) (b), $x$ is an extreme point of $B\left(L_{\Phi}^{0}\right)$. Let $\left(x_{n}\right)$ and $\left(y_{n}\right)$ be sequences in $L_{\Phi}^{0}$ such that $\left\|x_{n}\right\|_{\Phi}^{0} \rightarrow 1$ and $\left\|y_{n}\right\|_{\Phi}^{0} \rightarrow 1$ as $n \rightarrow \infty$ and $x_{n}+y_{n}=2 x$ for any $n \in \mathbb{N}$. We need to prove that $\left\|x_{n}-x\right\|_{\Phi}^{0} \rightarrow 0$ as $n \rightarrow \infty$. The proof requires the consideration of few cases separately.

Case $1^{0}$. Assume that $K\left(x_{n}\right) \neq \emptyset$ and $K\left(y_{n}\right) \neq \emptyset$ for any $n \in \mathbb{N}$ and $l=$ $\max \left\{\sup _{n} k_{n}, \sup _{n} h_{n}\right\}<\infty$ for some $k_{n} \in K\left(x_{n}\right)$ and $h_{n} \in K\left(y_{n}\right) \quad(n \in \mathbb{N})$. Then the sequence $\left(\frac{2 k_{n} h_{n}}{k_{n}+h_{n}}\right)$ is bounded. Assume without loss of generality (passing to a subsequence, if necessary) that $\lim _{n \rightarrow \infty} \frac{2 k_{n} h_{n}}{k_{n}+h_{n}}=h$. Then, in view of the Fatou lemma, we get

$$
\begin{aligned}
1 & =\|x\|_{\Phi}^{0} \\
& \leq \frac{1}{h}\left(1+I_{\Phi}(h x)\right) \\
& \leq \liminf _{n \rightarrow \infty} \frac{k_{n}+h_{n}}{2 k_{n} h_{n}}\left(1+I_{\Phi}\left(\frac{2 k_{n} h_{n}}{k_{n}+h_{n}} x\right)\right) \\
& \leq \liminf _{n \rightarrow \infty} \frac{k_{n}+h_{n}}{2 k_{n} h_{n}}\left(1+I_{\Phi}\left(\frac{h_{n}}{k_{n}+h_{n}}\left(k_{n} x_{n}\right)+\frac{k_{n}}{k_{n}+h_{n}}\left(h_{n} y_{n}\right)\right)\right) \\
& \leq \liminf _{n \rightarrow \infty} \frac{k_{n}+h_{n}}{2 k_{n} h_{n}}\left(1+\frac{h_{n}}{k_{n}+h_{n}} I_{\Phi}\left(k_{n} x_{n}\right)+\frac{k_{n}}{k_{n}+h_{n}} I_{\Phi}\left(h_{n} y_{n}\right)\right) \\
& =\liminf _{n \rightarrow \infty} \frac{1}{2}\left(\frac{1}{k_{n}}+\frac{1}{h_{n}}+\frac{1}{k_{n}} I_{\Phi}\left(k_{n} x_{n}\right)+\frac{1}{h_{n}} I_{\Phi}\left(h_{n} y_{n}\right)\right) \\
& =\liminf _{n \rightarrow \infty} \frac{1}{2}\left(\frac{1}{k_{n}}\left(1+I_{\Phi}\left(k_{n} x_{n}\right)\right)+\frac{1}{h_{n}}\left(1+I_{\Phi}\left(h_{n} y_{n}\right)\right)\right) \\
& =\liminf _{n \rightarrow \infty} \frac{1}{2}\left(\left\|x_{n}\right\|_{\Phi}^{0}+\left\|y_{n}\right\|_{\Phi}^{0}\right) \\
& =1 .
\end{aligned}
$$

Consequently, $h \in K(x)$. Since $K(x)=\{k\}$, we get $h=k$, i.e. $\lim _{n \rightarrow \infty} \frac{2 k_{n} h_{n}}{k_{n}+h_{n}}$ $=k$.

Next, we will show that $k_{n} x_{n}-h_{n} y_{n} \rightarrow 0$ in measure. Assume that this is not true. Then there exists $\varepsilon_{0}>0$ and $\delta_{0}>0$ such that $\mu\left(E_{n}\right) \geq \varepsilon_{0}$ for $n$ large enough, where

$$
E_{n}=\left\{t \in T:\left|k_{n} x_{n}(t)-h_{n} y_{n}(t)\right| \geq \delta_{0}\right\}
$$

Since $\frac{2 k_{n} h_{n}}{k_{n}+h_{n}} \rightarrow k$ as $n \rightarrow \infty$ and $x_{n}+y_{n}=2 x$ for any $n \in \mathbb{N}$, we conclude that

$$
\frac{k_{n} h_{n}}{k_{n}+h_{n}}\left(x_{n}+y_{n}\right)=\frac{2 k_{n} h_{n}}{k_{n}+h_{n}} x \rightarrow k x
$$


$\mu$-a.e. in $T$. Assume first that the measure of $T$ is finite. Then $\frac{k_{n} h_{n}}{k_{n}+h_{n}}\left(x_{n}+\right.$ $\left.y_{n}\right) \rightarrow k x$ in measure. Consequently, if

$$
A_{n}=\left\{t \in T: \frac{k_{n} h_{n}}{k_{n}+h_{n}}\left(x_{n}(t)+y_{n}(t)\right) \notin\left[k x(t)-\delta_{0}, k x(t)+\delta_{0}\right]\right\},
$$

then $\mu\left(A_{n}\right)<\frac{\varepsilon_{0}}{4}$ for $n$ large enough. Since $\bar{k}=\sup _{n} k_{n}<\infty$ and $L=$ $\sup _{n}\left\|x_{n}\right\|_{\Phi}^{0}<\infty$, we get

$$
\sup _{n} I_{\Phi}\left(k_{n} x_{n}\right)=\sup _{n}\left[k_{n}\left\|x_{n}\right\|_{\Phi}^{0}-1\right] \leq \bar{k} L-1=: M<\infty .
$$

Therefore, defining

$$
B_{n}=\left\{t \in T: k_{n}\left|x_{n}(t)\right|>d\right\}
$$

where $d>0$, we get for any $n \in \mathbb{N}$

$$
M>I_{\Phi}\left(k_{n} x_{n} \chi_{B_{n}}\right) \geq \Phi(d) \mu\left(B_{n}\right)
$$

whence $\mu\left(B_{n}\right) \leq \frac{M}{\Phi(d)}$. So there is $d>0$ such that $\mu\left(B_{n}\right)<\frac{\varepsilon_{0}}{4}$ for every $n \in \mathbb{N}$. Analogously, we can prove that $\mu\left(C_{n}\right)<\frac{\varepsilon_{0}}{4}$ for any $n \in \mathbb{N}$ if

$$
C_{n}=\left\{t \in T: h_{n}\left|y_{n}(t)\right|>d\right\}
$$

with $d>0$ large enough. Define

$$
\begin{aligned}
F_{n}= & \left\{t \in T:\left|k_{n} x_{n}(t)-h_{n} y_{n}(t)\right| \geq \delta_{0}, k_{n}\left|x_{n}(t)\right| \leq d, h_{n}\left|y_{n}(t)\right| \leq d\right\} \\
& \cap A_{n}^{\prime} .
\end{aligned}
$$

Then, for every $n \in \mathbb{N}$,

$$
\begin{aligned}
\mu\left(F_{n}\right) & \geq \mu\left(E_{n}\right)-\left(\mu\left(A_{n}\right)+\mu\left(B_{n}\right)+\mu\left(C_{n}\right)\right) \\
& >\varepsilon_{0}-\left(\frac{\varepsilon_{0}}{4}+\frac{\varepsilon_{0}}{4}+\frac{\varepsilon_{0}}{4}\right) \\
& =\frac{\varepsilon_{0}}{4} .
\end{aligned}
$$

Now, we will show that $k_{n} x_{n}(t)$ and $h_{n} y_{n}(t)$ are on different sides of $k x(t)$ for $t \in F_{n}$ and $n$ large enough. Notice that, for any $n \in \mathbb{N}$ and for all $t \in F_{n}$,

$$
\begin{aligned}
\frac{2 k_{n} h_{n}}{k_{n}+h_{n}}|x(t)| & =\left|\frac{2 k_{n} h_{n}}{k_{n}+h_{n}} x(t)\right| \\
& =\left|\frac{k_{n} h_{n}}{k_{n}+h_{n}}\left(x_{n}(t)+y_{n}(t)\right)\right| \\
& =\left|\frac{h_{n}}{k_{n}+h_{n}} k_{n} x_{n}(t)+\frac{k_{n}}{k_{n}+h_{n}} h_{n} y_{n}(t)\right| \\
& \leq \frac{h_{n}}{k_{n}+h_{n}}\left|k_{n} x_{n}(t)\right|+\frac{k_{n}}{k_{n}+h_{n}}\left|h_{n} y_{n}(t)\right| \\
& \leq \frac{h_{n}}{k_{n}+h_{n}} d+\frac{k_{n}}{k_{n}+h_{n}} d \\
& =d .
\end{aligned}
$$


804 Y. Cui et. al.

Since $\liminf n_{n \rightarrow \infty} k_{n} \geq 1$ and $\liminf _{n \rightarrow \infty} h_{n} \geq 1$, there exists $n_{1} \in \mathbb{N}$ such that $\frac{2 k_{n} h_{n}}{k_{n}+h_{n}} \geq \frac{3}{4}$ for $n>n_{1}$. Consequently,

$$
|x(t)| \leq \frac{4}{3} d
$$

for $n>n_{1}$. By $\lim _{n \rightarrow \infty} \frac{2 k_{n} h_{n}}{k_{n}+h_{n}}=k$ there is $n_{2}$ such that $\left|\frac{2 k_{n} h_{n}}{k_{n}+h_{n}}-k\right|<\frac{\delta_{0}}{4(1+\bar{k}) d}$ for every $n>n_{2}$. In view of (2) we get

$$
\begin{aligned}
& \left|\frac{h_{n}}{k_{n}+h_{n}} k_{n} x_{n}(t)+\frac{k_{n}}{k_{n}+h_{n}} h_{n} y_{n}(t)-k x(t)\right| \\
& \quad=\left|\frac{k_{n} h_{n}}{k_{n}+h_{n}}\left(x_{n}(t)+y_{n}(t)\right)-k x(t)\right| \\
& \quad=\left|\frac{2 k_{n} h_{n}}{k_{n}+h_{n}} x(t)-k x(t)\right| \\
& \quad=\left|\frac{2 k_{n} h_{n}}{k_{n}+h_{n}}-k\right||x(t)| \\
& \quad \leq \frac{\delta_{0}}{4(1+\bar{k}) d} \frac{4}{3} d \\
& \quad=\frac{\delta_{0}}{3(1+\bar{k})}
\end{aligned}
$$

for any $t \in F_{n}$ and $n>n_{0}=\max \left\{n_{1}, n_{2}\right\}$.

On the other hand, for any $t \in F_{n}$ and $n>n_{0}$ we have

$$
\begin{aligned}
& \left|\frac{k_{n} h_{n}}{k_{n}+h_{n}}\left(x_{n}(t)+y_{n}(t)\right)-h_{n} y_{n}(t)\right| \\
& =\left|\frac{h_{n}}{k_{n}+h_{n}} k_{n} x_{n}(t)+\frac{k_{n}}{k_{n}+h_{n}} h_{n} y_{n}(t)-h_{n} y_{n}(t)\right| \\
& =\left|\frac{h_{n}}{k_{n}+h_{n}} k_{n} x_{n}(t)+\left(\frac{k_{n}}{k_{n}+h_{n}}-1\right) h_{n} y_{n}(t)\right| \\
& =\left|\frac{h_{n}}{k_{n}+h_{n}} k_{n} x_{n}(t)-\frac{h_{n}}{k_{n}+h_{n}} h_{n} y_{n}(t)\right| \\
& =\left|\frac{h_{n}}{k_{n}+h_{n}}\left(k_{n} x_{n}(t)-h_{n} y_{n}(t)\right)\right| \\
& =\frac{h_{n}}{k_{n}+h_{n}}\left|\left(k_{n} x_{n}(t)-h_{n} y_{n}(t)\right)\right| \\
& \geq \frac{h_{n}}{\bar{k}+h_{n}} \delta_{0} \\
& \geq \frac{\frac{3}{4} \delta_{0}}{\bar{k}+\frac{3}{4}} \\
& >\frac{3}{4} \frac{\delta_{0}}{\bar{k}+1}
\end{aligned}
$$


because $\bar{k}=\sup _{n}\left\{k_{n}\right\}$ and the function $\frac{x}{\bar{k}+x}$ is increasing on $\left[\frac{3}{4}, \infty\right)$. Analogously, we can get

$$
\left|\frac{k_{n} h_{n}}{k_{n}+h_{n}}\left(x_{n}(t)+y_{n}(t)\right)-k_{n} x_{n}(t)\right| \geq \frac{3 \delta_{0}}{4(1+\bar{k})}
$$

for any $t \in F_{n}$ and $n>n_{0}$. Therefore, for any $t \in F_{n}$ and $n$ large enough, the distance between the point $\frac{k_{n} h_{n}}{k_{n}+h_{n}}\left(x_{n}(t)+y_{n}(t)\right)$ and each of the endpoints $k_{n} x_{n}(t)$ and $h_{n} y_{n}(t)$ of the interval is larger than $\frac{3 \delta_{0}}{4(1+\bar{k})}$, but the distance of this point from $k x(t)$ is less than $\frac{\delta_{0}}{3(1+k)}$. Thus $k_{n} x_{n}(t)$ and $h_{n} y_{n}(t)$ are on different sides of $k x(t)$ and

$$
\min \left\{\left|k_{n} x_{n}(t)-k x(t)\right|,\left|h_{n} y_{n}(t)-k x(t)\right|\right\}>\frac{\delta_{0}}{3(1+\bar{k})}
$$

for $t \in F_{n}$ and $n \geq n_{0}$. By the fact that $k x(t) \in S C(\Phi)$ for $\mu$-a.e. $t \in T$ we have

$$
\max \left\{\Phi\left(k_{n} x_{n}(t)\right), \Phi\left(h_{n} y_{n}(t)\right)\right\}>\Phi\left(a(\Phi)+\frac{\delta_{0}}{3(1+\bar{k})}\right)
$$

for $t \in F_{n}$ and $n \geq n_{0}$. Moreover,

$$
\begin{aligned}
& \Phi\left(\frac{h_{n}}{h_{n}+k_{n}}\left(k_{n} x_{n}(t)\right)+\frac{k_{n}}{h_{n}+k_{n}}\left(h_{n} y_{n}(t)\right)\right) \\
& \quad \quad<\frac{h_{n}}{h_{n}+k_{n}} \Phi\left(k_{n} x_{n}(t)\right)+\frac{k_{n}}{h_{n}+k_{n}} \Phi\left(h_{n} y_{n}(t)\right)
\end{aligned}
$$

for $n$ large enough and $t \in F_{n}$. Since the sequences $\left(k_{n}\right)$ and $\left(h_{n}\right)$ are bounded, there exists $\delta_{1}>0$ such that

$$
\begin{aligned}
& \Phi\left(\frac{h_{n}}{h_{n}+k_{n}}\left(k_{n} x_{n}(t)\right)+\frac{k_{n}}{h_{n}+k_{n}}\left(h_{n} y_{n}(t)\right)\right) \\
& \quad \quad<\left(1-\delta_{1}\right)\left\{\frac{h_{n}}{h_{n}+k_{n}} \Phi\left(k_{n} x_{n}(t)\right)+\frac{k_{n}}{h_{n}+k_{n}} \Phi\left(h_{n} y_{n}(t)\right)\right\}
\end{aligned}
$$

for $n$ large enough and $t \in F_{n}$. Hence

$$
\begin{aligned}
2 \leq & \left\|x_{n}+y_{n}\right\|_{\Phi}^{0} \\
\leq & \liminf _{n \rightarrow \infty} \frac{k_{n}+h_{n}}{k_{n} h_{n}}\left(1+I_{\Phi}\left(\frac{k_{n} h_{n}}{k_{n}+h_{n}}\left(x_{n}+y_{n}\right)\right)\right) \\
\leq & \liminf _{n \rightarrow \infty} \frac{k_{n}+h_{n}}{k_{n} h_{n}}\left[1+\int_{T \backslash F_{n}} \Phi\left(\frac{k_{n} h_{n}}{k_{n}+h_{n}}\left(x_{n}(t)+y_{n}(t)\right)\right) d \mu\right. \\
& \left.+\int_{F_{n}} \Phi\left(\frac{k_{n} h_{n}}{k_{n}+h_{n}}\left(x_{n}(t)+y_{n}(t)\right)\right) d \mu\right]
\end{aligned}
$$




$$
\begin{aligned}
\leq & \liminf _{n \rightarrow \infty}\left[\frac{1}{k_{n}}\left(1+\int_{T} \Phi\left(k_{n} x_{n}(t)\right) d \mu\right)+\frac{1}{h_{n}}\left(1+\int_{T} \Phi\left(h_{n} y_{n}(t)\right) d \mu\right)\right. \\
& \left.-\delta_{1} \int_{F_{n}}\left(\frac{h_{n}}{h_{n}+k_{n}} \Phi\left(k_{n} x_{n}(t)\right)+\frac{k_{n}}{h_{n}+k_{n}} \Phi\left(h_{n} y_{n}(t)\right)\right) d \mu\right] \\
\leq & \liminf _{n \rightarrow \infty}\left(2-\delta_{1} \bar{\delta} \int_{F_{n}}\left(\Phi\left(k_{n} x_{n}(t)\right)+\Phi\left(h_{n} y_{n}(t)\right)\right) d \mu\right) \\
\leq & \liminf _{n \rightarrow \infty}\left(2-\delta_{1} \bar{\delta} \int_{F_{n}} \max \left\{\Phi\left(k_{n} x_{n}(t)\right), \Phi\left(h_{n} y_{n}(t)\right)\right\} d \mu\right) \\
\leq & \liminf _{n \rightarrow \infty}\left[2-\delta_{1} \bar{\delta} \Phi\left(a(\Phi)+\frac{\delta_{0}}{3(1+\bar{k})}\right) \mu\left(F_{n}\right)\right] \\
\leq & 2-\frac{\delta_{1} \bar{\delta} \varepsilon_{0}}{4} \Phi\left(a(\Phi)+\frac{\delta_{0}}{3(1+\bar{k})}\right)
\end{aligned}
$$

where

$$
\bar{\delta}=\min \left(\inf _{n} \frac{2 h_{n}}{h_{n}+k_{n}}, \inf _{n} \frac{2 k_{n}}{h_{n}+k_{n}}\right) .
$$

The obtained contradiction shows that $k_{n} x_{n}-h_{n} y_{n} \rightarrow 0$ in measure when $\mu(T)<\infty$.

Assume now that $\mu(T)=\infty$. Since $\frac{2 k_{n} h_{n}}{h_{n}+k_{n}} \rightarrow k$, we conclude that

$$
\frac{k_{n} h_{n}}{h_{n}+k_{n}}\left(x_{n}(t)+y_{n}(t)\right)=\frac{2 k_{n} h_{n}}{h_{n}+k_{n}} x(t) \rightarrow k x(t)
$$

$\mu$-a.e. in $T$. Since $x_{n}+y_{n}$ is equal to the fixed function $2 x$ for any $n \in \mathbb{N}$, we can prove that $\frac{k_{n} h_{n}}{h_{n}+k_{n}}\left(x_{n}+y_{n}\right) \stackrel{\mu}{\longrightarrow} k x$ also when $\mu(T)=\infty$.

Next, we define in the same way as in the case when $\mu(T)<\infty$ the sets $F_{n}, A_{n}, B_{n}, C_{n}$ and $F_{n}$ obtaining that $\mu\left(F_{n}\right) \geq \frac{\varepsilon_{0}}{4}$ for $n$ large enough. Consequently, using this same argumentation as in the case when $\mu(T)<\infty$, we get that $k_{n} x_{n}-h_{n} y_{n} \stackrel{\mu}{\longrightarrow} 0$. Since $\frac{k_{n} h_{n}}{h_{n}+k_{n}}\left(x_{n}+y_{n}\right)$ is a convex combination of $k_{n} x_{n}$ and $h_{n} y_{n}$, so $\frac{k_{n} h_{n}}{h_{n}+k_{n}}\left(x_{n}(t)+y_{n}(t)\right)$ is in the interval $\left[k_{n} x_{n}(t), h_{n} y_{n}(t)\right]$ or $\left[h_{n} y_{n}(t), k_{n} x_{n}(t)\right]$. Therefore,

$$
\frac{k_{n} h_{n}}{h_{n}+k_{n}}\left(x_{n}+y_{n}\right)-k_{n} x_{n} \stackrel{\mu}{\longrightarrow} 0,
$$

that is $\frac{2 k_{n} h_{n}}{h_{n}+k_{n}} x-k_{n} x_{n} \stackrel{\mu}{\longrightarrow} 0$. The last condition and the fact that $\frac{2 k_{n} h_{n}}{h_{n}+k_{n}} \rightarrow k$ as $n \rightarrow \infty$ yield $k_{n} x_{n}-k x \stackrel{\mu}{\longrightarrow} 0$. The sequence $\left(k_{n}\right)$ is bounded, so we may assume (passing to a subsequence if necessary) that $k_{n} \rightarrow k^{\prime}$ as $n \rightarrow$ $\infty$ for some $k^{\prime}>0$. Since the sequence $\left(\left\|x_{n}\right\|_{\Phi}^{0}\right)$ is bounded, we have that 
$k_{n} x_{n}-k^{\prime} x_{n} \rightarrow 0$ in norm, whence $k_{n} x_{n}-k^{\prime} x_{n} \stackrel{\mu}{\longrightarrow} 0$. Combining this with $k_{n} x_{n} \stackrel{\mu}{\longrightarrow} k x$, we get $k^{\prime} x_{n} \stackrel{\mu}{\longrightarrow} k x$, that is

$$
\frac{k^{\prime}}{k} x_{n} \stackrel{\mu}{\longrightarrow} x
$$

Therefore, by the Fatou property of the norm $\|\cdot\|_{\Phi}^{0}$ (see [15]),

$$
1=\|x\|_{\Phi}^{0} \leq \liminf _{n \rightarrow \infty} \frac{k^{\prime}}{k}\left\|x_{n}\right\|_{\Phi}^{0}=\frac{k^{\prime}}{k} .
$$

Consequently, $k^{\prime} \geq k$.

We may assume without loss of generality (passing to a subsequence if necessary) that $h_{n} \rightarrow h^{\prime}$. We can prove in the same way as the inequality $k^{\prime} \geq k$ has been proved that $h^{\prime} \geq k$. Assume without loss of generality that $k^{\prime} \geq h^{\prime}$. By the Fatou Lemma we conclude that $\frac{2 k^{\prime} h^{\prime}}{h^{\prime}+k^{\prime}} \in K(x)=\{k\}$. Hence $\frac{2 k^{\prime} h^{\prime}}{h^{\prime}+k^{\prime}}=k$ and we claim that this yields $h^{\prime}=k^{\prime}=k$. Assume for the contrary that $k^{\prime}>k$. The function $f(k)=\frac{2 h^{\prime} k}{h^{\prime}+k}$ is strictly increasing on $(0, \infty)$, whence $k^{\prime}>k$ implies $\frac{2 h^{\prime} k}{h^{\prime}+k}<\frac{2 h^{\prime} k^{\prime}}{h^{\prime}+k^{\prime}}=k$, and consequently $\frac{2 h^{\prime}}{h^{\prime}+k}<1$, which contradicts the inequality $h^{\prime} \geq k$. This contradiction proves that $k^{\prime}=k$. Therefore, the equality $\frac{2 h^{\prime} k^{\prime}}{h^{\prime}+k^{\prime}}=k^{\prime}$ yields $\frac{2 h^{\prime}}{h^{\prime}+k}=1$, whence we get also $h^{\prime}=k$. So the claim is proved.

Therefore, by (3), $x_{n} \stackrel{\mu}{\longrightarrow} x$. Moreover, $\left\|x_{n}\right\|_{\Phi}^{0} \rightarrow\|x\|_{\Phi}^{0}$. Assumption (c) implies that $L_{\Phi}^{0}$ has the Kadec-Klee property with respect to the global convergence in measure (see [7]). Consequently, $\left\|x_{n}-x\right\|_{\Phi}^{0} \rightarrow 0$ and $\left\|y_{n}-x\right\|_{\Phi}^{0} \rightarrow 0$ as $n \rightarrow \infty$. This finishes the proof of Case 1 .

Case $2^{0}$. Assume the assumptions from Case $1^{0}$ do not hold. There are sequences $\left(k_{n}^{\prime}\right)$ and $\left(h_{n}^{\prime}\right)$ of positive numbers such that

$$
\begin{aligned}
\left\|x_{n}\right\|_{\Phi}^{0} & \geq \frac{1}{k_{n}^{\prime}}\left(1+I_{\Phi}\left(k_{n}^{\prime} x_{n}\right)\right)-\frac{1}{n} \\
\left\|y_{n}\right\|_{\Phi}^{0} & \geq \frac{1}{h_{n}^{\prime}}\left(1+I_{\Phi}\left(h_{n}^{\prime} y_{n}\right)\right)-\frac{1}{n}
\end{aligned}
$$

for all $n \in \mathbb{N}$. We do not know if the sequences $\left(k_{n}^{\prime}\right)$ and $\left(h_{n}^{\prime}\right)$ are bounded, so we will consider the sequences $\left(x_{n}^{\prime}\right)$ and $\left(y_{n}^{\prime}\right)$, where $x_{n}^{\prime}=\frac{1}{2}\left(x_{n}+x\right)$ and $y_{n}^{\prime}=\frac{1}{2}\left(y_{n}+x\right)$ in place of $\left(x_{n}\right)$ and $\left(y_{n}\right)$, because $\left\|x_{n}-x\right\|_{\Phi}^{0} \rightarrow 0$ and $\left\|y_{n}-x\right\|_{\Phi}^{0} \rightarrow 0$ as $n \rightarrow \infty$ if and only if $\left\|x_{n}^{\prime}-x\right\|_{\Phi}^{0} \rightarrow 0$ and $\left\|y_{n}^{\prime}-x\right\|_{\Phi}^{0} \rightarrow 0$ as $n \rightarrow \infty$, respectively. Moreover,

$$
\left\|\frac{1}{2}\left(x_{n}+x\right)\right\|_{\Phi}^{0} \leq \frac{1}{2}\left(\left\|x_{n}\right\|_{\Phi}^{0}+\|x\|_{\Phi}^{0}\right)
$$


for every $n \in \mathbb{N}$. Hence $\lim \sup _{n \rightarrow \infty}\left\|\frac{x_{n}+x}{2}\right\|_{\Phi}^{0} \leq 1$. In the same way we can prove that $\lim \sup _{n \rightarrow \infty}\left\|\frac{y_{n}+x}{2}\right\|_{\Phi}^{0} \leq 1$. Since $\frac{x_{n}+x}{2}+\frac{y_{n}+x}{2}=2 x$ for all $n \in \mathbb{N}$, we conclude that $\liminf _{n \rightarrow \infty}\left\|\frac{x_{n}+x}{2}\right\|_{\Phi}^{0} \geq 1$ and $\liminf _{n \rightarrow \infty}\left\|\frac{y_{n}+x}{2}\right\|_{\Phi}^{0} \geq 1$. Consequently, $\left\|\frac{x_{n}+x}{2}\right\|_{\Phi}^{0} \rightarrow 1$ and $\left\|\frac{y_{n}+x}{2}\right\|_{\Phi}^{0} \rightarrow 1$ as $n \rightarrow \infty$.

Define

$$
w_{n}=\frac{2 k_{n}^{\prime} k}{k_{n}^{\prime}+k} \quad \text { and } \quad v_{n}=\frac{2 h_{n}^{\prime} k}{h_{n}^{\prime}+k} .
$$

The sequences $\left(w_{n}\right)$ and $\left(v_{n}\right)$ are bounded. Moreover,

$$
\begin{aligned}
\left\|\frac{x_{n}+x}{2}\right\|_{\Phi}^{0} & \leq \frac{1}{w_{n}}\left(1+I_{\Phi}\left(w_{n} \frac{x_{n}+x}{2}\right)\right) \\
& =\frac{k_{n}^{\prime}+k}{2 k_{n}^{\prime} k}\left(1+I_{\Phi}\left(\frac{k_{n}^{\prime} k}{k_{n}^{\prime}+k}\left(x_{n}+x\right)\right)\right) \\
& =\frac{k_{n}^{\prime}+k}{2 k_{n}^{\prime} k}\left(1+I_{\Phi}\left(\frac{k}{k_{n}^{\prime}+k}\left(k_{n}^{\prime} x_{n}\right)+\frac{k_{n}^{\prime}}{k_{n}^{\prime}+k}(k x)\right)\right) \\
& \leq \frac{1}{2}\left\{\frac{1}{k_{n}^{\prime}}\left(1+I_{\Phi}\left(k_{n}^{\prime} x_{n}\right)\right)+\frac{1}{k}\left(1+I_{\Phi}(k x)\right)\right\} \\
& \leq \frac{1}{2}\left\{\left\|x_{n}\right\|_{\Phi}^{0}+\frac{1}{n}+\|x\|_{\Phi}^{0}\right\} \\
& \rightarrow 1 \quad(n \rightarrow \infty)
\end{aligned}
$$

whence it follows that

$$
\frac{k_{n}^{\prime}+k}{2 k_{n}^{\prime} k}\left(1+I_{\Phi}\left(\frac{k_{n}^{\prime} k}{k_{n}^{\prime}+k}\left(x_{n}+x\right)\right)\right) \rightarrow 1 \quad(n \rightarrow \infty) .
$$

Analogously,

$$
\frac{h_{n}^{\prime}+k}{2 h_{n}^{\prime} k}\left(1+I_{\Phi}\left(\frac{h_{n}^{\prime} k}{h_{n}^{\prime}+k}\left(y_{n}+x\right)\right)\right) \rightarrow 1 \quad(n \rightarrow \infty) .
$$

Therefore, we can prove in the same way as in Case $1^{0}$ that $\left\|x_{n}^{\prime}-x\right\|_{\Phi}^{0} \rightarrow 0$ and $\left\|y_{n}^{\prime}-x\right\|_{\Phi}^{0} \rightarrow 0$ as $n \rightarrow \infty$.

Case $3^{0}$. Suppose that assumptions (a) and (b) are satisfied. We will show that if additionally $\Phi(b(\Phi))<\infty$, then $x \in S\left(L_{\Phi}^{0}\right)$ such that $k|x(t)|=b(\Phi)$ for $\mu$-a.e. $t \in T$ is a strongly extreme point of $B\left(L_{\Phi}^{0}\right)$. Namely, assume that $\left(x_{n}\right)$ and $\left(y_{n}\right)$ are sequences such that $\left\|x_{n}\right\|_{\Phi}^{0} \rightarrow 1$ and $\left\|y_{n}\right\|_{\Phi}^{0} \rightarrow 1$ as $n \rightarrow \infty$ and $x_{n}+y_{n}=2 x$. We have $K\left(x_{n}\right) \neq \emptyset$ and $K\left(y_{n}\right) \neq \emptyset$ by $b(\Phi)<\infty$. Passing to the sequences $\left(\frac{x_{n}+x}{2}\right)$ and $\left(\frac{y_{n}+y}{2}\right)$ in place of $\left(x_{n}\right)$ and $\left(y_{n}\right)$ if necessary, we may assume that the sequences $\left(k_{n}\right)$ and $\left(h_{n}\right)$, where $k_{n} \in K\left(x_{n}\right)$ and $h_{n} \in K\left(y_{n}\right)$ for any $n \in \mathbb{N}$, are bounded. We may assume without loss of 
generality that $\left(k_{n}\right)$ and $\left(h_{n}\right)$ are convergent. Their limits must be equal to $k$ as it follows from the proof of Case $1^{0}$. From the equalities

$$
\begin{gathered}
I_{\Phi}\left(k_{n} x_{n}\right)=k_{n}-1, \quad I_{\Phi}\left(h_{n} y_{n}\right)=h_{n}-1 \\
\lim _{n \rightarrow \infty} k_{n}=\lim _{n \rightarrow \infty} h_{n}=k, \quad I_{\Phi}(k x)=k-1
\end{gathered}
$$

we have

$$
\begin{aligned}
I_{\Phi}\left(k_{n} x_{n}\right) & \rightarrow I_{\Phi}(k x) \\
I_{\Phi}\left(h_{n} y_{n}\right) & \rightarrow I_{\Phi}(k x)
\end{aligned} \quad(n \rightarrow \infty)
$$

Therefore, we may assume without loss of generality that $I_{\Phi}\left(k_{n} x_{n}\right)<\infty$ and $I_{\Phi}\left(h_{n} y_{n}\right)<\infty$ for all $n \in \mathbb{N}$. Consequently, $k_{n}\left|x_{n}(t)\right| \leq b(\Phi)$ and $h_{n}\left|y_{n}(t)\right| \leq$ $b(\Phi)$ for $\mu$-a.e. $t \in T$ and for any $n \in \mathbb{N}$. Hence and from $\lim _{n \rightarrow \infty} k_{n}=k=$ $\lim _{n \rightarrow \infty} h_{n}$ it follows that $k\left|x_{n}(t)\right| \leq b(\Phi)$ and $k\left|y_{n}(t)\right| \leq b(\Phi)$ for $\mu$-a.e. $t \in T$ and all $n \in \mathbb{N}$. Therefore, for $\mu$-a.e. $t \in T$ and for any $n \in \mathbb{N}$, we have

$$
2 b(\Phi)=2 k|x(t)|=k\left|x_{n}(t)+y_{n}(t)\right| \leq k\left(\left|x_{n}(t)\right|+\left|y_{n}(t)\right|\right) \leq 2 b(\Phi),
$$

so $\left|x_{n}(t)\right|=\left|y_{n}(t)\right|=|x(t)|$. Consequently, $x_{n}=y_{n}=x$. Therefore $x$ is a strongly extreme point. This finishes the proof

Remark 2. Assume that $\mu(T)=\infty, x \in S\left(L_{\Phi}^{0}\right), K(x)=\{k\}$ for some $0<k<\infty$ and $k|x(t)|=b(\Phi)$ for $\mu$-a.e. $t \in T$. Then condition (c) from Theorem 2 is satisfied and $a(\Phi)=b(\Phi)$. In fact, if $a(\Phi)<b(\Phi)$, then $\Phi(b(\Phi))>0$ and $I_{\Phi}(k x)=\infty$ by $\mu(T)=\infty$, which contradicts the assumption $K(x)=\{k\}$. The equality $a(\Phi)=b(\Phi)$ yields that $L_{\Phi}^{0}=L^{\infty}$ and there is $L>0$ such that $\|x\|_{\Phi}^{0}=L\|x\|_{\infty}$ for any $x \in L_{\Phi}^{0}$. Moreover, the equalities $a(\Phi)=b(\Phi)=k|x(t)|$ for $\mu$-a.e. $t \in T$ imply by Theorem 2 that $x$ is a strongly extreme point.

Our results cover among others classical Banach spaces like the space $L^{\infty}$, the interpolation spaces $L^{1}+L^{\infty}$ and $L^{1} \cap L^{\infty}$ as well as the spaces $L^{p} \cap L^{\infty}$ with $1<p<\infty$.

\section{Some applications}

We start with the following well known result.

Corollary 4. In the space $L^{\infty}$ extreme points and strongly extreme points of $B\left(L^{\infty}\right)$ coincide. The only such points are functions $x \in L^{0}(\mu)$ such that $|x(t)|=1$ for $\mu$-a.e. $t \in T$.

Proof. It is easy to see that $L^{\infty}$ is the Orlicz space (with equality of norms) $L_{\Phi_{\infty}}^{0}$ where

$$
\Phi_{\infty}(u)= \begin{cases}0 & \text { for } u \in[-1,1] \\ +\infty & \text { otherwise }\end{cases}
$$


Obviously, $a\left(\Phi_{\infty}\right)=b\left(\Phi_{\infty}\right)=1$. The only points of strict convexity of $\Phi$ are +1 and -1 . Note that for any $x \in L^{0}(\mu)$ with $|x(t)|=1$ for $\mu$-a.e. $t \in T$ we have $K(x)=\{1\}$. Consequently, conditions (a) and (b) from Theorem 1 are satisfied. These facts, in view of Theorem 1 , show that the only extreme points are the ones mentioned above. Moreover, Theorem 2 yields that all those points are strongly extreme, which finishes the proof

Now we can apply Theorems 1 and 2 to the classical interpolation space $L^{1}+L^{\infty}$ equipped with the norm

$$
\|x\|_{L^{1}+L^{\infty}}=\inf \left\{\|y\|_{1}+\|z\|_{\infty}: y+z=x, y \in L^{1}, z \in L^{\infty}\right\}
$$

(see $[1,20])$. Criteria for extreme points of the unit ball of this space are known (see $[10,13,25])$, but criteria for strongly extreme points of its unit ball were still unknown. On the base of our Theorems 1 and 2 we can easily deduce not only criteria for extreme points but also for strongly extreme points of the unit ball of the space $L^{1}+L^{\infty}$.

Corollary 5. Let $x \in S\left(L^{1}+L^{\infty}\right)$. Then the following statements are equivalent:

(a) $x$ is an extreme point of $B\left(L^{1}+L^{\infty}\right)$

(b) $\mu(T)>1$ and $|x(t)|=1$ for $\mu$-a.e. $t \in T$

(c) $x$ is a strongly extreme point of $B\left(L^{1}+L^{\infty}\right)$.

If $\mu(T) \leq 1$, then the set of extreme points of the unit ball $B\left(L^{1}+L^{\infty}\right)$ is empty.

Proof. It is known (see [12]) that $L^{1}+L^{\infty}$ is an Orlicz space generated by the Orlicz function $\Phi_{\infty, 1}$ defined by the formula $\Phi_{\infty, 1}(u)=\max \{0,|u|-1\}$. Moreover, $\|\cdot\|_{L^{1}+L^{\infty}}=\|\cdot\|_{\Phi_{\infty}, 1}^{0}$. Suppose that $x \in S\left(L^{1}+L^{\infty}\right)$.

(a) $\Rightarrow(\mathrm{b})$. Let $x$ be an extreme point of $B\left(L^{1}+L^{\infty}\right)$. Then, by Theorem 1 , there is $k_{0} \geq 1$ such that $k_{0} x(t) \in S C\left(\Phi_{\infty, 1}\right)$ for $\mu$-a.e. $t \in T$ and $K(x)=\left\{k_{0}\right\}$. But $S C(\Phi)=\{-1,1\}$, so $\left|k_{0} x(t)\right|=1$ for $\mu$-a.e. $t \in T$. If $k_{0}>1$, then

$$
\|x\|_{L^{1}+L^{\infty}}=\frac{1}{k_{0}}\left(1+\int_{T} \Phi_{\infty, 1}\left(k_{0} x(t)\right) d \mu\right)=\frac{1}{k_{0}}<1
$$

which means that $x$ cannot be an element of the unit sphere $S\left(L^{1}+L^{\infty}\right)$. Hence it must be $k_{0}=1$ and consequently $|x(t)|=1$ for $\mu$-a.e. $t \in T$. Now it is enough to verify conditions under which statement (a) of Theorem 1 for the function $x$ is satisfied. Computing the norm of $x$ using the Amemiya formula 
we get

$$
\begin{aligned}
\|x\|_{L^{1}+L^{\infty}} & =\inf _{k>0} \frac{1}{k}\left(1+I_{\Phi_{\infty}, 1}\left(k \chi_{T}\right)\right) \\
& =\inf _{k>0} \frac{1}{k}\left(1+\int_{T} \Phi_{\infty, 1}(k) d \mu\right) \\
& =\min \left\{\inf _{k \in(0,1]} \frac{1}{k}, \inf _{k \in(1, \infty)}\left[\frac{1}{k}+\left(1-\frac{1}{k}\right) \mu(T)\right]\right\} \\
& =\min \left\{1, \inf _{k \in(1, \infty)}\left[\frac{1}{k}(1-\mu(T))+\mu(T)\right]\right\} .
\end{aligned}
$$

Now we will consider three cases separately.

If $\mu(T)<1$, then $\frac{1}{k}(1-\mu(T))+\mu(T)$ is a decreasing function of the variable $k$. Consequently,

$$
\inf _{k \in(1, \infty)}\left[\frac{1}{k}(1-\mu(T))+\mu(T)\right]=\lim _{k \rightarrow \infty}\left[\frac{1}{k}(1-\mu(T))+\mu(T)\right]=\mu(T) .
$$

Hence $\|x\|_{L^{1}+L^{\infty}}=\mu(T)<1$, so $x \notin S\left(L^{1}+L^{\infty}\right)$, whence it follows that $x$ is not an extreme point of $B\left(L^{1}+L^{\infty}\right)$. (It is easy to notice that $K(x)=\emptyset$ in this case).

If $\mu(T)=1$, then $\|x\|_{L^{1}+L^{\infty}}=1$, but $K(x)=[1, \infty)$ and condition (a) of Theorem 1 is not satisfied. Therefore, by Theorem $1, x$ cannot be an extreme point of $B\left(L^{1}+L^{\infty}\right)$.

If $\mu(T)>1$, then $\frac{1}{k}(1-\mu(T))+\mu(T)$ is an increasing and continuous function of the variable $k$. Hence

$$
\inf _{k \in(1, \infty)}\left[\frac{1}{k}(1-\mu(T))+\mu(T)\right]=\lim _{k \rightarrow 1}\left[\frac{1}{k}(1-\mu(T))+\mu(T)\right]=\mu(T) .
$$

Consequently, $\|x\|_{L^{1}+L^{\infty}}=\min \{1, \mu(T)\}=1$ and $K(x)=\{1\}$. Hence condition (a) of Theorem 1 is satisfied. Therefore $\mu(T)>1$ and $|x(t)|=1$ for $\mu$-a.e. $t \in T$, i.e. (b) holds true.

(b) $\Rightarrow$ (c). Let $\mu(T)>1$ and $|x(t)|=1$ for $\mu$-a.e. $t \in T$. By the above construction, it is obvious that conditions (a) and (b) of Theorem 2 are satisfied. We observe that $\Phi_{\infty, 1} \in \Delta_{2}(\infty)$ and $a\left(\Phi_{\infty, 1}\right)=1>0$. This means that condition (c) of Theorem 2 is also satisfied. Hence, by Theorem 2, $x$ is a strongly extreme point.

$(c) \Rightarrow(a)$. This implication is trivial, because in any Banach space every strongly extreme point of the unit ball is extreme.

By the implication $(\mathrm{a}) \Rightarrow(\mathrm{b})$, it follows immediately that in the case $\mu(T) \leq 1$ the unit ball $B\left(L^{1}+L^{\infty}\right)$ has no extreme points. This finishes the proof 
Consider the space $L^{1} \cap L^{\infty}$ equipped with the norm

$$
\|\cdot\|_{L^{1} \cap L^{\infty}}=\|\cdot\|_{L^{1}}+\|\cdot\|_{L^{\infty}} .
$$

The extreme points of $B\left(L^{1} \cap L^{\infty}\right)$ are characterized by Hudzik, Kamińska and Mastyło in [13]. We can also easily get their criteria using Theorem 1. Moreover, applying Theorem 2 we can find a characterization of strongly extreme points of $B\left(L^{1} \cap L^{\infty}\right)$. In contrast to the spaces $L^{\infty}$ and $L^{1}+L^{\infty}$, the extreme point of $B\left(L^{1} \cap L^{\infty}\right)$ need not be strongly extreme, which follows from the following

Corollary 6. A point $x \in S\left(L^{1} \cap L^{\infty}\right)$ is extreme if and only if it is of the form $|x|=k^{-1} \chi_{A}$, where $\mu(A)<\infty$ and $k=1+\mu(A)$.

The unit ball $B\left(L^{1} \cap L^{\infty}\right)$ has strongly extreme points if and only if $\mu(T)<$ $\infty$. The only strongly extreme points of $B\left(L^{1} \cap L^{\infty}\right)$ are the extreme points corresponding to $A=T$.

Proof. Since $L^{1} \cap L^{\infty}$ is the Orlicz space $L_{\Phi_{1, \infty}}^{0}$ with equality of norms, where $\Phi_{1, \infty}(u)=\max \left\{|u|, \Phi_{\infty}(u)\right\}$ and $\Phi_{\infty}$ is defined in the proof of Corollary 4 (see [13]), we can apply Theorems 1 and 2 .

If $|x|=(1+\mu(A))^{-1} \chi_{A}$ with $\mu(A)<\infty$, then $K(x)=\{1+\mu(A)\}$. In fact, since

$$
\inf _{k>0} \frac{1}{k}\left(1+I_{\Phi_{1, \infty}}\left(k(1+\mu(A))^{-1} \chi_{A}\right)\right)=\inf _{0<k \leq 1+\mu(A)}\left(\frac{1}{k}+\frac{\mu(A)}{1+\mu(A)}\right),
$$

$k=1+\mu(A)$ is the only number for which the infimum is attainable and $x \in S\left(L^{1} \cap L^{\infty}\right)$. Obviously, $(1+\mu(A)) x(t) \in S C \Phi_{1, \infty}$ for $\mu$-a.e. $t \in T$ because $S C \Phi_{1, \infty}=\{-1,0,1\}$. Hence, by Theorem 1, $x$ is an extreme point of $B\left(L^{1} \cap L^{\infty}\right)$.

Now assume that $x \in S\left(L^{1} \cap L^{\infty}\right)$ is an extreme point. Then, by Theorem $1, K(x)=\{k\}$ and $k x(t) \in S C \Phi_{1, \infty}=\{-1,0,1\}$ for $\mu$-a.e. $t \in T$. Consequently, $k|x(t)|=1$ for $\mu$-a.e. $t \in \operatorname{supp} x=A$. The only $k>0$ which satisfies that condition and the equality $\frac{1}{k}\left(1+I_{\Phi_{1, \infty}}(k x)\right)=1$ is $k=1+\mu(A)$. Indeed, the last equality is equivalent to $I_{\Phi_{1, \infty}}(k x)=k-1$. Hence, by $k|x(t)|=1$ for $\mu$-a.e. $t \in A$, we have $\Phi_{1, \infty}(1) \mu(A)=k-1$, i.e. $k=1+\mu(A)$.

It remains to give a proof of the criteria for strongly extreme points. Since $\Phi_{1, \infty}\left(b\left(\Phi_{1, \infty}\right)\right)=1$, by Theorem 2 , an extreme point $x$ of $B\left(L_{\Phi_{1, \infty}}^{0}\right)$ can be strongly extreme only in the case when $k|x(t)|=b\left(\Phi_{1, \infty}\right)=1$ for $\mu$-a.e. $t \in T$, where $\{k\}=K(x)$. As we has already shown, $k=1+\mu(\operatorname{supp} x)$. Since $k \in K(x)$ yields $I_{\Phi_{1, \infty}}(k x)<\infty$, we conclude that it must be $\mu(\operatorname{supp} x)<\infty$, i.e. $\mu(T)<\infty$. Consequently, the corollary is proved 
Consider now for $1<p<\infty$ the space $L^{p} \cap L^{\infty}$ equipped with the norm proposed in [15]:

$$
\|x\|_{L^{p} \cap L^{\infty}}= \begin{cases}\beta(x)^{p-1}\|x\|_{L^{p}}+\|x\|_{L^{\infty}} & \text { if } \beta(x) \leq\left(\frac{q}{p}\right)^{\frac{1}{p}} \\ p^{\frac{1}{p}} q^{\frac{1}{q}}\|x\|_{L^{p}} & \text { if } \beta(x)>\left(\frac{q}{p}\right)^{\frac{1}{p}}\end{cases}
$$

where $\beta(x)=\frac{\|x\|_{L^{p}}}{\|x\|_{L^{\infty}}}$ for $x \neq 0$ and $\frac{1}{p}+\frac{1}{q}=1$.

Corollary 7. The space $L^{p} \cap L^{\infty}$ is strictly convex. If $\mu(T) \leq \frac{q}{p}$, then a point $x \in S\left(L^{p} \cap L^{\infty}\right)$ is strongly extreme if and only if $|x|=(1+\mu(T))^{-1} \chi_{T}$. In the case when $\mu(T)>\frac{q}{p}$, the unit ball $B\left(L^{p} \cap L^{\infty}\right)$ has no strongly extreme points.

Proof. It is proved in [15] that $L^{p} \cap L^{\infty}$ equipped with the above norm is the Orlicz space $L_{\Phi_{p, \infty}}^{0}$, where $\Phi_{p, \infty}(u)=\max \left\{|u|^{p}, \Phi_{\infty}(u)\right\}$. Note that $S C\left(\Phi_{p, \infty}\right)=[-1,1]$. Let $x \in S\left(L^{p} \cap L^{\infty}\right)$. Then, by the fact that $I_{\Phi_{p, \infty}}(k x)=$ $\infty$ for $k>\frac{1}{\|x\|_{L^{\infty}}}$, we have

$$
\begin{aligned}
1 & =\|x\|_{L^{p} \cap L^{\infty}} \\
& =\inf _{k>0} \frac{1}{k}\left(1+I_{\Phi_{p, \infty}}(k x)\right) \\
& =\inf _{0<k \leq \frac{1}{\|x\|_{L^{\infty}}}} \frac{1}{k}\left(1+\int_{T}(k|x|)^{p} d \mu\right) \\
& =\inf _{0<k \leq \frac{1}{\|x\|_{L^{\infty}}}} \frac{1}{k}\left(1+k^{p}\|x\|_{L^{p}}^{p}\right) .
\end{aligned}
$$

Consider a real function $f$ of variable $k>0$ defined by the formula

$$
f(k)=\frac{1}{k}\left(1+a k^{p}\right)
$$

where $a>0$ is a real constant. The function $f$ is differentiable on $(0, \infty)$. Its derivative is of the form

$$
f^{\prime}(k)=-\frac{1}{k^{2}}+a(p-1) k^{p-2} .
$$

It is easy to calculate that $f^{\prime}(k)<0$ if and only if $k<\left(\frac{q}{a p}\right)^{1 / p}, f^{\prime}(k)=0$ for $k=\left(\frac{q}{a p}\right)^{1 / p}$ and $f^{\prime}(k)>0$ for $k>\left(\frac{q}{a p}\right)^{1 / p}$. This means that $f(k)$ is decreasing on $\left(0,\left(\frac{q}{a p}\right)^{1 / p}\right)$ and increasing on $\left(\left(\frac{q}{a p}\right)^{1 / p}, \infty\right)$. Hence, taking $a=\|x\|_{L^{p}}^{p}$, we consider two cases. 
If

$$
\frac{1}{\|x\|_{L^{\infty}}} \leq \frac{1}{\|x\|_{L^{p}}}\left(\frac{q}{p}\right)^{\frac{1}{p}}
$$

then the only number at which the infimum is attainable is $k_{0}=\frac{1}{\|x\|_{L^{\infty}}}$.

If

$$
\frac{1}{\|x\|_{L^{\infty}}}>\frac{1}{\|x\|_{L^{p}}}\left(\frac{q}{p}\right)^{\frac{1}{p}}
$$

then the infimum is attainable only at $k_{0}=\left(\frac{1}{\|x\|_{L^{p}}}\left(\frac{q}{p}\right)^{1 / p}\right.$.

Thus, $K(x)=\left\{k_{0}\right\}$ and $k_{0}|x(t)| \in S C\left(\Phi_{p, \infty}\right)$ for $\mu$-a.e. $t \in T$. Therefore, by Theorem $1, x$ is an extreme point of $B\left(L^{p} \cap L^{\infty}\right)$. This proves that the space $L^{p} \cap L^{\infty}$ is strictly convex.

Note that $b\left(\Phi_{p, \infty}\right)=1$ and $\Phi_{p, \infty}\left(b\left(\Phi_{p, \infty}\right)\right)=1$. Consequently, by (c) of Theorem 2, any strongly extreme point $x$ is characterized by the equalities $\|x\|_{L^{p} \cap L^{\infty}}=1$ and $k_{0}|x(t)|=1$ for $\mu$-a.e. $t \in T$, where $k_{0}$ is from (a) of Theorem 2. Hence $I_{\Phi_{p, \infty}}\left(\chi_{T}\right)=k_{0}-1$. Taking into account the definition of $\Phi_{p, \infty}$, we get $\mu(T)<\infty$ and $k_{0}=1+\mu(T)$. Consequently, $|x|=(1+$ $\mu(T))^{-1} \chi_{T}$. It is not difficult to prove that $\|x\|_{L^{p} \cap L^{\infty}}<1$ if $\mu(T)>\frac{q}{p}$ and $\|x\|_{L^{p} \cap L^{\infty}}=1$ if $\mu(T) \leq \frac{q}{p}$. Thus, when $\mu(T) \leq \frac{q}{p}$, each strongly extreme point of the unit ball is of the form $|x(t)|=\frac{1}{1+\mu(T)}$ for $\mu$-a.e. $t \in T$. The proof is finished

By the space of finite (that is order continuous) elements we will mean the subspace $E_{\Phi}^{0}$ of $L_{\Phi}^{0}$ defined by

$$
E_{\Phi}^{0}=\left\{x \in L_{\Phi}^{0}: I_{\Phi}(\lambda x)<\infty \text { for every } \lambda>0\right\} .
$$

The space $E_{\Phi}^{0}$ equipped with the norm topology induced from $L_{\Phi}^{0}$ is a closed subspace of $L_{\Phi}^{0}$. Hence $E_{\Phi}^{0}$ is a Banach space. It is worth to describe its set of extreme points because it plays an important role in studying the problems concerning duals of Orlicz spaces. Note that $E_{\Phi}^{0}=\{0\}$ provided $b(\Phi)<\infty$, so there is no sense of considering properties of $E_{\Phi}^{0}$ in this case.

Corollary 8. Let $b(\Phi)=\infty$. Then $\operatorname{ext} B\left(E_{\Phi}^{0}\right)=E_{\Phi}^{0} \cap \operatorname{ext} B\left(L_{\Phi}^{0}\right)$.

Proof. Since $E_{\Phi}^{0}$ is embedded isometrically into $L_{\Phi}^{0}$, the inclusion

$$
E_{\Phi}^{0} \cap \operatorname{ext} B\left(L_{\Phi}^{0}\right) \subset \operatorname{ext} B\left(E_{\Phi}^{0}\right)
$$

holds true. If $x \in \operatorname{ext} B\left(E_{\Phi}^{0}\right)$, then, repeating the proof the necessity of Theorem 1, we get conditions (a) and (b) of this theorem. Hence $x \in \operatorname{ext} B\left(L_{\Phi}^{0}\right)$. Consequently, ext $B\left(E_{\Phi}^{0}\right) \subset E_{\Phi}^{0} \cap \operatorname{ext} B\left(L_{\Phi}^{0}\right)$ and the proof is finished 
Corollary 9. Let $b(\Phi)=\infty$. If one of the conditions

(i) $I_{\Psi}\left(p \circ u_{0} \chi_{T}\right)<1$, where $u_{0}=\sup \{u \geq a(\Phi): u \in S C(\Phi)\}$

(ii) $S C(\Phi)=\{0\}$

(iii) $a(\Phi)>0$ and $\mu(T)=\infty$

is satisfied, then $\operatorname{ext} B\left(E_{\Phi}^{0}\right)=\emptyset$.

Proof. By Corollaries 3 and 8, if (i) or (ii) is satisfied, then ext $B\left(E_{\Phi}^{0}\right)=$ $\emptyset$. To prove the sufficiency of condition (iii), suppose ext $B\left(E_{\Phi}^{0}\right) \neq \emptyset$. Then by Corollary 8 and Theorem 1 there is $x_{0} \in S\left(E_{\Phi}^{0}\right)$ with exactly one $k_{0} \geq 1$ such that $\left\|x_{0}\right\|_{\Phi}^{0}=\frac{1}{k_{0}}\left(1+I_{\Phi}\left(k_{0} x_{0}\right)\right)$ and $k_{0} x_{0}(t) \in S C(\Phi)$ for $\mu$-a.e. $t \in T$. Let condition (iii) be satisfied. Since $a(\Phi)=\inf \{u \geq a(\Phi): u \in S C(\Phi)\}$, we have

$$
I_{\Phi}\left(2 k_{0} x_{0}\right) \geq I_{\Phi}(2 a(\Phi))=\Phi(2 a(\Phi)) \mu(T)=\infty
$$

i.e. $x_{0} \notin E_{\Phi}^{0}-$ contradiction. It shows that also in this case $\operatorname{ext} B\left(E_{\Phi}^{0}\right)=\emptyset$, which finishes the proof

Corollary 10. Assume $\Phi$ is an Orlicz function with $b(\Phi)=\infty$ and $x \in$ $S\left(E_{\Phi}^{0}\right)$. Then $x$ is a strongly extreme point of $B\left(E_{\Phi}^{0}\right)$ if and only if $x$ is an extreme point of $B\left(E_{\Phi}^{0}\right), \Phi \in \Delta_{2}(\infty)$ and at least one of the conditions

(i) $\mu(T)<\infty$

(ii) $\Phi \in \Delta_{2}(0)$

is satisfied.

Proof. Since $L_{\Phi}^{0}=E_{\Phi}^{0}$ provided $\Phi \in \Delta_{2}(\infty)$ and one of the conditions (i) or (ii) is satisfied, by Theorem 2 we conclude the sufficiency of the corollary. If $x$ is a strongly extreme point of $B\left(E_{\Phi}^{0}\right)$, then $x$ is an extreme point of $B\left(E_{\Phi}^{0}\right)$. By Corollary 4, we conclude that in the case $a(\Phi)>0$ and $\mu(T)=\infty$ also the set of strongly extreme points of $B\left(E_{\Phi}^{0}\right)$ is empty. Hence, excluding this situation and taking into account the assumption $b(\Phi)=\infty$, by Theorem 2 , we conclude the necessity of the assumptions

\section{References}

[1] Benett, C. and R. Sharpley: Interpolation of Operators. London et al.: Acad. Press 1988.

[2] Chen, S.: Geometry of Orlicz Spaces. Diss. Math. 356 (1996), 1 - 204.

[3] Chen, S., Cui, Y. and H. Hudzik: Isometric copies of $l^{1}$ and $l^{\infty}$ in Orlicz spaces equipped with the Orlicz norm. Proc. Amer. Math. Soc. (to appear). 
[4] Cui, Y., Hudzik, H., Nowak, M. and R. Płuciennik: Some geometric properties in Orlicz sequence spaces equipped with Orlicz norm. J. Convex Anal. 6 (1999), $91-113$.

[5] Cui, Y. and T. Wang: Strongly extreme points in Orlicz spaces (in Chinese). J. Math. 7 (1987), $335-340$.

[6] Diestel, J.: Sequences and Series in Banach Spaces (Graduate Texts in Mathematics: Vol. 92). New York et al.: Springer-Verlag 1984.

[7] Domínguez, T., Hudzik, H., López, G., Mastyło, M. and B. Sims: Complete characterizations of the Kadec-Klee properties in Orlicz spaces. Houston J. Math. (to appear).

[8] Giles, J. R. and W. B. Moors: Generic continuity of minimal set-valued mappings. J. Austral. Math. Soc. 63 (1997), 238 - 262.

[9] Grza̧ślewicz, R., Hudzik, H. and W. Kurc: Extreme and exposed points in Orlicz spaces. Canad. J. Math. 44 (1992), 505 - 515.

[10] Grzasślewicz, R. and H. Schaefer: On the isometries of $L^{1} \cap L^{\infty}[0, \infty)$ and $L^{1}+L^{\infty}[0, \infty)$. Indag. Math. 3 (1992), $173-178$.

[11] Hudzik, H.: Orlicz spaces without strongly extreme points and without H-points. Canad. Math. Bull. 36 (1993), 173 - 177.

[12] Hudzik, H.: On the distance from the subspace of order continuous elements in $L^{1}+L^{\infty}$. Funct. et Appr. 26 (1997), $157-163$.

[13] Hudzik, H., Kamińska, A. and M. Mastyło: Local geometry of $L^{1} \cap L^{\infty}$ and $L^{1}+L^{\infty}$. Arch. Math. 68 (1997), $159-168$.

[14] Hudzik, H., Kurc, W. and M. Wisła: Strongly extreme points in Orlicz function space. J. Math. Anal. \& Appl. 189 (1995), 651 - 670.

[15] Hudzik, H. and L. Maligranda: Amemiya norm equals Orlicz norm in general. Indag. Math. (N.S.) 11 (2000), 573 - 585.

[16] Hudzik, H. and M. Wisła: On extreme points of Orlicz spaces with Orlicz norm. Collect. Math. 44 (1993), 135 - 146.

[17] Kamińska, A.: On uniform convexity of Orlicz spaces. Ind. Math. A85 (1982), $473-749$.

[18] Kamińska, A.: Strict convexity of Musielak-Orlicz sequence spaces with Orlicz nor. J. Funct. Anal. 50 (1983), 285 - 305.

[19] Krasnoselskii, M. A. and Ya. B. Rutickii: Convex Functions and Orlicz spaces. Groningen: Nordhoff 1961.

[20] Krein, S. G., Petunin, Ju. I. and E. M. Semenov: Interpolation of Linear Operators (AMS Trans. Math. Mon.: Vol. 54). Providence, R.I.: Amer. Math. Soc. 1982 .

[21] Maligranda, L.: Orlicz Spaces and Interpolation (Seminários de Matemática: Vol. 5). Campinas (Brazil): Univ. Estadual de Campinas 1989.

[22] Musielak, J.: Orlicz Spaces and Modular Spaces. Lect. Notes Math. 1034 (1983), $1-222$. 
[23] Płuciennik, R., Wang Tingfu and Zhang Yonglin: H-points and denting points in Orlicz spaces. Comment. Math. 33 (1993), 135 - 151.

[24] Rao, M. M. and Z. D. Ren: Theory of Orlicz Spaces. New York et al.: Marcel Dekker 1991.

[25] Schaefer, H.: On convex hulls. Arch. Math. 58 (1992), 160 - 163.

[26] Smith, M. A.: A curious generalization of local uniform rotundity. Comment. Math. Univ. Carolin. 25 (1984), 659 - 665.

[27] Wu, C., Wang, T., Chen, S. and Y. Wang: Geometric Theory of Orlicz Spaces (in Chinese). Harbin (China): Harbin Univ. of Techn. Press 1986.

Received 29.01.2003 